\title{
Screening of Hematopoietic Small Molecules \\ Facilitates the Establishment of a Chemically Defined Hsc Expansion Medium With Enhanced Performance
}

\section{Esra Albayrak}

Yeditepe University: Yeditepe Universitesi

\section{Sezer Akgol}

Yeditepe University: Yeditepe Universitesi

\section{Raife Dilek Turan}

Acıbadem Mehmet Ali Aydınlar Üniversitesi: Acibadem Universitesi

\section{Fatih Kocabas ( $\nabla$ fatih.kocabas@yeditepe.edu.tr )}

Yeditepe University https://orcid.org/0000-0001-8096-6056

\section{Research Article}

Keywords: Hematopoietic stem cells, HSCT, expansion, and quiescence

Posted Date: March 2nd, 2021

DOI: https://doi.org/10.21203/rs.3.rs-254465/v1

License: (c) (1) This work is licensed under a Creative Commons Attribution 4.0 International License. Read Full License 


\section{Abstract}

Hematopoietic stem cells (HSC), known for their ability to multipotent and self-renew, are often used in HSC transplantation for the treatment of hematological diseases and malignancies. Umbilical cord blood (UCB) and mobilized peripheral blood (mPB), which are alternatives to bone marrow (BM) for HSC transplantation, have reduced HSC. To address this restriction, the ex vivo expansion of HSCs is a highly promising therapeutic approach rather than the use of a double-cord blood unit that induces delays in hematopoietic recovery. We have previously shown that knockout of HSC quiescence genes could increase the HSC pool in vivo. Thus, we thought that targeting HSC quiescence regulators using small molecules could be used for ex vivo expansion of both mPB and UCB-HSC. The goal was to identify novel hematopoietic small molecules (HSMs) and their combinations, and to enhance performance of human HSC expansion medium. We identified and analyzed 35 possible HSMs targeting HSC quiescence factors. We assessed their impact on human HSPC activity, including expansion, quiescence, multilineage capacity, cycling capability and metabolism. We have also investigated their cytotoxic and genotoxic effects on human HSPCs. On the other hand, a transplantation study was performed on immunocompromised mice for the evaluation of the repopulation and engraftment capacities of the expanded cells. We observed that BML-260 and TAME molecules robustly increased both the MPB and UCB-HSPC content and activated the re-entry of HSCs into the cell cycle. Colony Forming Unit (CFU) assay confirmed their improved multilineage capacity. BML-260 proved safer for the viability of expanded cells based on cytotoxicity and genotoxicity assays. In addition, gene expression analysis showed that BML-260 and TAME molecules contributed to HSC expansion by modulating cell cycle kinetics, including p27, Skp2 and Cdh1. In conjunction with these in vitro results, we have observed that BML-260-expanded HSCs had a strong hematopoietic reconstitution capacity. After the determination of the most effective molecule as BML-260, a comparative study of chemically defined media, including various supplements, was analyzed in addition to the BML-260 molecule. These results from in vitro and xenotransplantation experiments have shown that BML-260 molecules can be used therapeutically for human HSC expansion and regulation of HSC activity. In addition, the medium composition found may be a novel platform for human HSC expansion to used in clinical trials.

\section{Highlights}

- We have studied 35 putative hematopoietic small molecules to make it possible to develop a chemically defined HSC expansion medium with better efficiency

- BML-260 and TAME treatments induced ex vivo expansion of both human mPB- and UCB-derived hematopoietic stem cells by targeting of quiescence factors.

- BML-260-expanded HSCs functionally reconstituted the entire hematopoiesis in LT-engraftment

- The screening of small hematopoietic molecules enabled development of a high-performance chemically based HSC expansion medium

\section{Introduction}

Hematopoietic stem cells are present in various human samples, including bone marrow, peripheral blood and umbilical cord blood, albeit low in the quantity. They reside in quiescence form by arresting at the cell cycle and entry to the G0 phase(1). They play an important role in the process of hematopoiesis, described as the formation of blood cells. HSCs are a regenerative medicine pupil because of their stem cell properties for the 
treatment of hematological malignancies and blood disorders. In despite of their limited self-renewal capacity, HSCs are pupil of the regenerative medicine because of their stem cell properties for the treatment of hematological malignancies and blood disorders. Millions of people suffered from hematological malignancies, blood disorders and congenital metabolic defects are treated with HSC transplantation (HSCT) every year. Bone marrow and peripheral blood are the primary reservoirs of HSCT, but lack of the (human leukocyte antigen) HLAmatched donor has been dispatched to identify alternate sources or processes. Thus, umbilical cord blood (UCB) has been used as an alternative. The main limitation of UCB HSC transplantation is that HSC number is not enough for high incidence of hematopoietic recovery post effective transplantation. The clinical studies showed that minimum cell dose of $3 \times 10^{7}$ nucleated cells $/ \mathrm{kg}$ is required for successful engraftment. To overcome this, two different UCB unit have been used but, this approach implies some problems about engraftment capacity and recovery rate $(2,3)$.

Ex vivo expansion of HSCs is major therapeutic solution to overcome the limited cell numbers and enhanced hematopoietic reconstitution in clinical use for various hematological disorders. Until today, the diverse combinations of various cytokines and growth factors have been used to support HSC expansion. However, these culture and expansion protocols did not provide maintenance of HSC self-renewal and remained incapable for delay of differentiation $(4,5)$. Small molecules are the chemicals that have low molecular weight $(<900$ Dalton), easily diffuse from cell membrane and non-immunogenic. They are extensively used for their ability on regulation of the stem cell fate, proliferation, self-renewal, differentiation and cell death. They are also very valuable tools to improve therapeutic approaches because of their features including lower cost, facility of manipulation, and control $(6,7)$. Thus, small molecules could be therapeutically used to enhance HSC number instead of usage of double cord blood unit resulting in increased risk of GVHD and thus, reduced hematopoietic recovery. Recent studies have shown that small molecules become very important for clinical grade HSC expansion along with improvement of computational methods $(8,9)$. Current studies focusing on the promotion of self-renewal, inhibition of differentiation, inhibition of apoptosis, and promotion of homing of HSCs have shown that some of identified small molecules including the aryl hydrocarbon antagonist StemRegenin 1 (SR1), pyrimidoindole derivative UM171, prostaglandin derivative $\mathrm{PGE}_{2}$, histone deacetylase inhibitor Nicotinamide (NAM) and Cu chelator tetraethylenepentamine (TEPA) have been investigated in clinical trials(6).

In recent years, several cell culture methods including culture systems, supplementation of cytokines, growth factors and/or small molecules have been utilized for HSC expansion. Chao-Ling Yao et. al., carried out an investigation about the strategy for development of chemically defined serum-free medium based on adding appropriate supplements and/or reinforcement molecules into basal medium such as DMEM, RPMI, and IMDM(10). Utilization of this approach in clinical studies is required for production of HSC products under good manufacturing practices (GMP) conditions which control production of medical products depending on quality standards, determined usage and license requirements in accordance with requirement of product specifications(11). Several GMP-grade products such as StemPro ${ }^{\mathrm{TW}}$, Stemline, HSC-Brew, and CellGenix expansion medium that related to HSC expansion have been developed by ThermoScientific, Sigma, Miltenyi Biotech and CellGenix companies, respectively. However, there is still for improvement in terms of content as well HSC expansion capabilities of those products. In addition, their composition is not always accessible to scientific community, which slows processes in the innovation of novel HSC expansion platforms.

In this study, we screened previously uncharacterized 26 putative hematopoietic small molecules (HSMs) along with previously reported ones on MPB and UCB HSCs to determine the effective HSMs and/or HSM cocktail for 
development of chemically defined medium, which will enhance human HSC expansion. We found that several new small molecules are promising for both human mPB- and UCB-derived HSC ex vivo expansion through modulation of cell cycle kinetics. Xenotransplantation study on SCID mice confirmed the repopulation capacity of HSM expanded cells in long-term engraftment. On the other hand, the chemically defined HSC medium composition including RPMI 1640 basal medium supplemented with cytokine and HSM combinations shown to be promising for development of chemically defined human HSC expansion medium.

\section{Materials And Methods}

\subsection{Small molecules and samples}

Small molecules were dissolved as $10 \mathrm{mM}$ stock concentrations (Supplementary Table 1). Dimethyl sulfoxide (DMSO) (0.25\%) was used as a negative control (DMSO, Santa Cruz Biotech, Cat. No:Sc-3590329).

\subsection{Isolation and culture of MNCs and CD34 + cells}

UCB and mPB mononuclear cells were isolated by using Ficoll-Paque (HistopaqueTM, Sigma, Cat.No:10831) density gradient centrifugation $(1,083 \mathrm{~g} / \mathrm{ml})$ method in 24 hours post obtaining as we have done previousy $(12)$. Firstly, the blood samples were diluted as 1:1 proportion in a $15 \mathrm{ml}$ falcon tube with Dulbecco's PhosphateBuffered Saline (DPBS, Thermofisher Scientific, Cat. No:14190144) and mixed gently by inverting. Diluted blood samples were added to equal volume Ficoll-Paque of non-diluted blood and centrifuged at $3000 \mathrm{rpm}$ for 15 minutes without brake. After centrifugation, upper phase including serum was removed and cloudy interphase, also known as buffy coat, was transferred to new falcon tube. The transferred cells were washed with $3 x$ volume of DPBS and mixed by gentle inverting. The cells were centrifuged at $1500 \mathrm{rpm}$ for 5 minutes with brake and then, the supernatant was removed. The pellet was re-suspended in $10 \mathrm{ml}$ DPBS and mononuclear cells (MNCs) were counted by hemocytometer to perform the appropriate experiments. Besides, to obtain CD $34+$ cells from MNCs, the MNCs were labelled with DAPI (4', 6-Diamidino-2-Phenylindole, Dihydrochloride) (Thermofisher Scientific, Cat.No:D1306) and PE-conjugated CD34, (Biolegend, Cat.No:343506) according to the manufacturer's manual post isolation of the MNCs from fresh samples. The CD34 + cells were separated from the labelled cells by Biorad Cell Sorter (Biorad S3E Cell Sorter, Cat. No:24L4D). UCB- and mPB-MNCs and CD34 + cells were cultured in the medium which consists of Roswell Park Memorial Institute (RPMI) 1640 Medium (Gibco, Cat.No:11875085) supplemented with 2 percent FBS (Gibco, Cat.No:12483020) 1 percent PSA (10,000 units/ml penicillin and 10,000 ug/ml streptomycin and $25 \mu \mathrm{g} / \mathrm{mL}$ of Amphotericin B; Gibco, Cat.No:15240062) and human cytokine cocktail (StemSpan ${ }^{\text {TM }}$ CC100, Stemcell Technologies, Cat.No:02690).

\subsection{Immunophenotyping}

The human UCB- and mPB-MNCs were seeded on 96 well plate at a density of 30,000 cells per well in the medium. The seeded cells were treated with each of 35 putative HSMs at $0.1,1$ and $10 \mu \mathrm{M}$ concentrations. The cells used as control were treated with dimethyl sulfoxide (DMSO) $(0.25 \%)$. The treated cells were incubated in 7 days at $37^{\circ} \mathrm{C}$, in $5 \% \mathrm{CO}_{2}$ and under normoxic conditions. The treated UCB- and mPB- MNCs were labelled with FITC-conjugated CD90, PE-conjugated CD34, PE-Cy7-conjugated CD38 (Biolegend, Cat. No:328107, 343506 and 303516; respectively) and APC-conjugated CD133 (eBioscience, Cat.No:17-1338-42) antibodies according to the manufacturer's manual (1:1000 dilution ratio). The HSC content of the labelled cells were analyzed by flow cytometry (Cytoflex S, Beckman, Cat.No:B47903). 


\subsection{Cell cycle and proliferation analysis}

Human CD34 + cells from UCB or mPB-MNC were sorted from MNC population by fluorescence activated cell sorting (FACS) after labelling with anti-human PE-conjugated CD34 as described above. The CD34 + cells were seeded in the HSC expansion medium at a density of 10,000 cells per well in 96 well-plate and treated with effective doses (shown in Supplementary Table 2) of the 2-APB, BML-260 and TAME molecules and 0.25\% DMSO control. After 4 days of the treatment, expanded HSCs were labelled with Hoechst 33342 (1 ng/sample; stock: $10 \mu \mathrm{g} / \mathrm{ml}$ ) (Sigma Aldrich, USA, Cat. No:14533) and incubated at $37^{\circ} \mathrm{C}$ and $5 \% \mathrm{CO} 2$ for 30 minutes, then stained with Pyronin Y (10ng/sample; stock:100 $\mathrm{g} / \mathrm{ml}$ ) (Sigma Aldrich, USA, Cat.No:P9172-1G) and incubated at $37^{\circ} \mathrm{C}$ and $5 \% \mathrm{CO} 2$ for 15 minutes. Following the staining, the cells were analyzed by flow cytometry compared to DMSO control as we have done previously(13). On the other hand, the human mPB-CD34 + were seeded on 30,000 cells per well in the expansion medium. The seeded cells were treated with the effective doses of BML260 and TAME molecules and $0.25 \%$ of DMSO. The treated cells were labelled with 5-(and-6) carboxyfluorescein succinimidyl ester (CFSE) (as $5 \mu \mathrm{M}$ final concentration for $15 \mathrm{~min}$. at $37^{\circ} \mathrm{C}$ ) (CFDA-SE, Stemcell Technologies, Cat.No:75003) and then, the labelling was stopped with 10\% FBS/PBS. The sample separated at day 0 was analyzed by flow cytometry. After 1 and 7 days of incubation time, the CFSE + cells in labelled cells were analyzed by flow cytometry.

\subsection{Colony Forming Unit (CFU) assays}

The human UCB-MNCs were seeded at 30,000 cells/well on 96-well plate and treated with the most effective doses (shown in Supplementary Table 2) of 2-APB, BML-260 and TAME molecules and 0.25\% of DMSO. After 7 days of expansion, the cells were harvested and counted. After that, the cells were plated in methylcellulosecontaining medium (MethoCult ${ }^{\mathrm{TM}}$ GF M3434, Stemcell Technologies, Cat.No:03444) at a density of 20,000 cells per well in 6-well plate, performed in triplicate. After 14 days, colonies were classified as CFU-GEMM, CFU-G/M, BFU-E and B cell colonies and counted by light microscopy as we have done previously(12).

\subsection{ALDH and ROS measurements}

Aldehyde dehydrogenase (ALDH) enzyme activity was measured on expanded MNCs derived from mPB post treatment with effective doses of 2-APB, BML-260 and TAME molecules and $0.25 \%$ of DMSO in three replicates according to manufacturer's protocol (Stemcell, Aldefluor Kit, Cat.No:01700). Briefly, 30,000 cells/well were collected and centrifuged at $300 \mathrm{~g}$ for $5 \mathrm{~min}$. The pellet was re-suspended in 200ul of ALDEFLUOR assay buffer. $1 \mu \mathrm{l}$ of ALDEFLUOR reagent was added to all samples. The cells were analyzed by flow cytometry following 45 minutes incubation at $37^{\circ} \mathrm{C}$. ALDEFLUOR ${ }^{\mathrm{TM}}$ Diethylaminobenzaldehyde (DEAB) reagent ( $1 \mu \mathrm{l}$ in $200 \mathrm{ul}$ sample) was added to control tube. The control tube was analyzed post labeling with ALDEFLUOR reagent following 4 hours of incubation with DEAB. The gating on FITC (fluorescein isothiocyanate) was set according to DEAB control. To analyze the Reactive Oxygen Species (ROS), the mPB-MNCs seeded at a density of 30,000 cells/well were treated with effective doses of 2-APB, BML-260 and TAME molecules and $0.25 \%$ of DMSO. Post 7 days of treatment, the ROS activity was measured on expanded HSPCs by flow cytometry post labeling of ROS with 2',7'-dichlorofluorescin diacetate (DCFDA) fluorogenic dye according to manufacturer's protocol (DCFDA / H2DCFDA - Cellular ROS Assay Kit, Abcam, Cat.No:ab113851). Briefly, the treated cells were stained with (20 $\mu \mathrm{M}$ working concentration) DCFDA for 30 min (without washing) and then, measured by flow cytometer with excitation / emission at $485 \mathrm{~nm} / 535 \mathrm{~nm} .50 \mu \mathrm{M}$ Tert-Butyl Hydrogen Peroxide (TBHP) was used as a positive control following dilution from $55 \mathrm{mM}$ stock solution. 


\subsection{Cytotoxicity, genotoxicity and viability analysis}

The sorted human mPB- CD34 + cells were seeded at a density of 10,000 cells/well on 96-well plate and treated with effective doses of 2-APB, BML-260 and TAME molecules. After 72 hours of the treatments, the cells were collected from 96-well plates and centrifuged at $1500 \mathrm{rpm}$ for 5 minutes. The pellets were re-suspended in $50 \mu \mathrm{l}$ of $1 \mathrm{X}$ binding buffer (BD Pharmingen, Cat. No:556570), then stained with 1ul of FITC Annexin V and incubated at room temperature for 15 minutes. Then $200 \mu \mathrm{l}$ X binding buffer was added onto the stained cells and labelled with 2 ul of PI (Propoidium lodide) according to the modified manufacturer's manual (eBioscience, Cat. No:888005-74). The stained cells were analyzed by flow cytometry compared to DMSO control (0.25\%). Genotoxicity analysis was assessed by using Apoptosis, DNA Damage and Cell Cycle Kit (BD, Cat. No:562253) post 7 days of treatments. Firstly, the mPB- MNCs (at least 100,000 cells / well) were treated with effective doses of p300/CBP Inhibitor VI, 2-APB, BML-260 and TAME molecules and $0.25 \%$ of DMSO. Utilization of this assay designed with the inclusion of fluorescent antibodies specific for incorporated BrdU-PC5.5, phosphorylated H2AX-APC, and cleaved PARP-PE was required to antibody binding following the fixation and permeabilization of the cells according to manufacturer's protocol. To evaluate the viability of the HSM expanded cells, the human mPBMNCs were seeded on 96-well plate at a density of 30,000 cells per well in the expansion medium. The seeded cells were treated with the most effective doses of 2-APB, BML-260, TAME molecules and DMSO control. After 7 days of treatment, the viability of expanded cells was determined by flow cytometry following labeling of the treated cells with 7AAD (7-Amino-Actinomycin D) (1:1000 dilution) (BD, Cat.No:559925). The count and percentage were quantified.

\subsection{Gene expression analysis}

The gene expression of cylin-dependent kinase inhibitors (CDKIs), S-phase, homology directed repair (HDR) and target pathway-related genes were analyzed by Real-Time Polymerase Chain Reaction (RT-PCR). For that, the RNA isolation from mPB-MNCs were performed by Invitrogen PureLink RNA Mini Kit (Thermoscientific, Cat.No:12183025) according to the manufacturer's protocol post 4 days of treatment with effective doses of BML-260 and TAME molecules and $0.25 \%$ of DMSO following culture of $2 \times 10^{6}$ cells per well on the 6 well plate. After the RNA isolation, RNAs were converted to cDNA by using ProtoScript ${ }^{\circledR}$ First Strand cDNA Synthesis Kit (NEB, Cat.No:E6300S). For quantitative PCR analysis, GoTaq® qPCR Master Mix (Promega, Cat.No:A6002) was used with predesigned primers (shown in Supplementary Table 3) obtained from databases (Gene Card etc.) and ordered from Sentegen Biotechnology, Turkey. qPCR reactions were conducted on LightCycler ${ }^{\circledR} 96$ Real-Time PCR system (Roche Health Caree, Cat.No:12953). Data were analyzed by using $\Delta \Delta C$ t method and $\beta$-Actin was used as a housekeeping gene to normalize the results.

\subsection{Repopulation and engraftment assays}

Human mPB-CD34 + cells isolated by FACS were transplanted to SCID mice expressing CD45.1 allele post 7 days of culture in HSC expansion medium including BML-260 and DMSO control. Short-term and long-term repopulation and engraftment analyzes were performed at 4- and 20-weeks time interval, respectively. Peripheral blood cells were just analyzed after 4-weeks, peripheral blood and bone marrow cells were collected and analyzed after 20-weeks for long-term immune reconstitution. Briefly, peripheral blood cells were isolated by lysis of red blood cells post taking of PB sample from SCID mice with retro-orbital bleeding method. These cells were labeled with anti-human CD45-FITC and anti-mouse CD45.1- PE (phycoerythrin) (Biolegend, Cat. No:110707) for short-term repopulation assay. Besides, these cells were analyzed for immune reconstitution post labeling with 
murine lymphoid antibodies; anti-Thy1.2-PE (eBioscience, Cat No. 12-0903-81), anti- B220-PE (Biolegend, Cat. No:103207) and myeloid antibodies; anti-Mac-1-PE (Biolegend, Cat. No:101207) and anti-Gra-1-PE (Biolegend, Cat. No:108407) along with anti-human CD45-FITC. In addition, long-term repopulation was assessed by analysis with human lineage antibodies; anti-human CD3-FITC (for T lymhocytes), CD19-PE (for B lymphocytes), CD33-PE (for myeloid cells). In addition to that, BM cells were collected by flushing of PBS in bone marrow following taking of femur and tibia bones from mice. Isolated cells were stained with human HSPC-specific antibodies; PE-conjugated CD34 (eBioscience, Cat. No:12-0349-42), (allophycocyanin) APC-conjugated CD133 (eBioscience, Cat. No:17-1338-42), and PE-Cyanine-conjugated CD38 (eBioscience, Cat. No:25-0389-42) antibodies, and murine HSPC-specific antibodies; c-Kit (CD117)-PE, CD34-FITC, Sca-1-PE-Cy7, and lineage cocktail-APC (BD StemFlow, Cat. No:560492) and then, analyzed by flow cytometry as we have done previously(14).

\subsection{Comparative analysis of chemically defined-medium compositions}

To determine the effective chemically-defined basal medium including appropriate supplementations, Roswell Park Memorial Institute Medium (RPMI 1640) and Iscove's Modified Dulbecco Medium (IMDM) basal mediums were prepared as different compositions with 5\% FBS, 10\% FBS, 20\% FBS and serum-free conditions. These medium compositions were prepared in two types; with and without insulin-transferrin-selenium mixture. $0.1 \mu \mathrm{M}$ concentration of BML-260 small molecule and STF cytokine combinations (SCF, TPO and FLT3) were common supplements for every medium compositions (shown in Table 1). Post culture of mPB-MNCs with these different medium compositions, HSPC content analysis was assessed by flow cytometry following the labeling of the expanded cells with human HSC surface markers as described in previous section.

Table 1

The chemically-defined HSC expansion medium compositions

\begin{tabular}{|lllll|}
\hline Supplements & FBS & $\begin{array}{l}\text { Cytokines } \\
(\mathrm{STF})\end{array}$ & $\begin{array}{l}\text { BML-260 } \\
(0.1 \mu \mathrm{M})\end{array}$ & $\begin{array}{l}\text { Insulin-Transferrin-Selenium } \\
(\mathrm{ITS})\end{array}$ \\
\hline RPMI/IMDM-1 & $\mathrm{SF}$ & + & + & - \\
\hline RPMI/IMDM-2 & $\mathrm{SF}$ & + & + & + \\
RPMI/IMDM-3 & $5 \%$ & + & + & - \\
\hline RPMI/IMDM-4 & $5 \%$ & + & + & + \\
RPMI/IMDM-5 & $10 \%$ & + & + & - \\
\hline RPMI/IMDM-6 & $10 \%$ & + & + & + \\
\hline RPMI/IMDM-7 & $20 \%$ & + & + & - \\
\hline RPMI/IMDM-8 & $20 \%$ & + & + & + \\
\hline
\end{tabular}

\subsection{Statistical Analysis}

The statistics were analyzed by Dunnett’s Multiple Comparison Test. p $₫ 0.05$ were considered statistically significant and $\mathrm{p} \otimes 0.01$ were measured more statistically significant. 


\section{Results}

\subsection{Identification of HSMs for ex vivo expansion of human HSPCs}

To develop an improved and novel small molecule-based human HSC expansion platform, we formed small molecule pool including 35 HSMs. To this end, we analyzed over 200 genes, which of knockout studies carried out in HSCs in vivo. We primarily found that 109 genes from 200 genes increased the cell cycle activity or proliferation capacity of HSCs following knockout of themselves and also, 57 of 109 genes was found to increase the expansion of stem cell pool more than 1,5-fold. After that, we selected 30 genes from 57 genes according to their availability as small molecule inhibitor in CHEMBL, PubChem and similar small molecule databases. 26 compounds of them have been procured. In addition to them, we decided to add 9 new compounds, which was found to be related to quiescence of cardiomyocytes depending on our cardiac regeneration studies based on the idea that HSCs display similar quiescence profile with cardiomyocytes $(15,16)$. Eventually, we have selected 35 putative HSM targeting 35 hematopoietic quiescence factors (Fig. 1), which 9 of them were shown to poses activity on previous studies (reviewed in (17)) and 26 of them have not been previously studied for small molecule induced ex vivo HSC expansion and were further tested in this study (Supplementary Table 1).

\subsection{Screening of HSMs on UCB and mPB-HSPCs showed that 2-APB, BML-260, TAME and MEIS1 inhibitors are promising new small molecules for human HSPC expansion}

To determine the effective HSMs among 35 putative molecules on human HSC expansion, small molecule screening was firstly assessed on UCB hematopoietic cells by flow cytometry analysis. The assay was performed post labeling of the HSM treated UCB-MNCs with human HSC characterization markers including CD34-PE, CD38-PC7, CD133-APC and ALDH-FITC post 7 days of treatment with $0.1,1$ and $10 \mu \mathrm{M}$ doses of all HSMs. HSPC analysis was evaluated with flow cytometry gating strategy (Fig. 2a) showed the changes in total cell count, CD34 + cell count, CD133 + cell ratio and ALDHhi cell count in comparison to DMSO $(0.25 \%)$ control samples. The findings showed that treatment with \#9, \#12,\#16, \#17, \#21, \#31, \#32, \#33, \#34 and \#35 numbered molecules increased the total cell count more than 2-fold (Fig. 2b). Remarkably, treatment with \#10, $\# 11, \# 14, \# 19, \# 23$ and \#33 numbered molecules increased the CD34 + cell count more than 4-fold, while treatments of $\# 3, \# 7, \# 8, \# 9, \# 12, \# 16, \# 17, \# 24, \# 26, \# 31, \# 32, \# 34$ and \#35 numbered molecules increased more than 2-fold. Interestingly, \#2 numbered molecule increased the CD34 + cell count more than 7-fold (Fig. 2c).

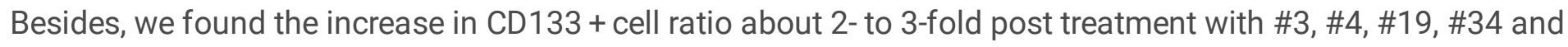
\#35 numbered molecules (Fig. 2d).

Then, we analyzed the HSM-treated cells depending on their ALDH enzyme activity which is known to be high in HSCs because of metabolically glycolysis pathway preference for energy production(16). We determined that

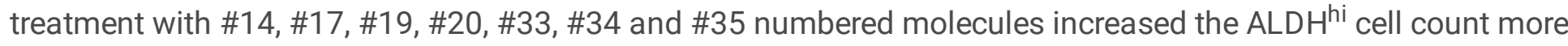
than 3-fold (Fig. 2e). All HSMs were assessed depending on their dose dependent effect on treated cells (Figure S1a-d). Taken together, we briefly determined that treatment with \#2 (p300/CBP Inhibitor VI), \#19 (2-APB), \#32 (BML-260), \#33 (TAME), \#34 (MEISi-1) and \#35 (MEISi-2) inhibitors among 35 HSMs highly induced enhancement of UCB-HSPC content compared to DMSO and previously tested molecules. Therefore, we selected 
these untested compounds in addition to the previously tested control compounds for the next experiments to investigate their role in human HSC expansion.

We did not only assess the effects of these HSMs on UCB hematopoietic cells but also, we assessed their effect on mobilized PB hematopoietic cells. We evaluated the profile of HSPC content post 7 days of treatment with 0.1, 1 and $10 \mu \mathrm{M}$ doses of HSMs selected on UCB experiment. We used the previously tested compound bpV(Hopic) as a positive control. HSPC analysis evaluated with flow cytometry gating strategy (Fig. 3a) indicated that different doses of HSMs were the most effective for HSPC expansion. We have recorded that p300/CBP Inhibitor VI, BML-260, TAME, MEISi-1 and MEISi-2 HSMs induced the increase in CD34+, CD34 + CD38and CD133 + cell populations of mPB-HSPC content more than 2-fold to 5-fold compared to DMSO and previously tested positive control compound (Fig. 3b-d). Intriguingly, p300/CBP Inhibitor VI molecule demonstrated higher increase in the \% CD34 + cells (Fig. 3b).

\subsection{BML-260 and TAME small molecules induced reentry of UCB- and mPB-HSC to cell cycle and enhanced multilineage potential}

Re-entry to cell cycle is a critical issue for expansion of HSCs characterized by cell cycle arrest resulting in cell quiescence during ex vivo procedures. Hence, we performed the cell cycle analysis post 4 days of treatment with the optimum doses of 2-APB, BML-260 and TAME molecules on mPB and UCB-CD34 + cells. We indicated that treatments with BML-260 and TAME molecules increased the UCB-CD34 + cell ratio in S/G2/M phase more than 5 -fold and decreased in the cell ratio in $G_{0}$ and $G_{1}$ phase compared to DMSO control (Fig. 4a). When we analyzed on mPB-CD34 + cells, we showed that treatments of BML-260 and TAME molecules significantly increased (approximately 5-fold) the $\mathrm{mPB}-\mathrm{CD} 34+$ cell ratio in $\mathrm{G}_{1}$ phase and reduced the cell ratio in $\mathrm{G}_{0}$ phase compared to DMSO control (Fig. 4b). Briefly, BML-260 and TAME induced re-entry to cell cycle from quiescent state of HSCs. Interestingly, treatment with 2-APB molecule triggered re-entry of UCB-CD34 + cells to cell cycle, but did not for mPB-CD34 + cells. Next, we performed the CFSE proliferation assay to confirm the mPB-CD34 + cell expansion. The CFSE fluorescence was measured on day $1 \& 7$ and compared to DMSO control with optimum doses of BML-260 and TAME molecules. We found that treatments of BML-260 and TAME molecules showed increased cycling cells at Day 1 and Day 7 (Figure S2).

To investigate the multilineage potential of HSM treated UCB-HSPCs, human colony forming unit assay was performed post 7 days of treatment with 2-APB, BML-260 and TAME compounds. We found an increase in the number of GEMM colonies post treatment with 2-APB, BML-260 and TAME compounds compared to DMSO control (Fig. 4c). Besides, number of GM colonies was also higher post treatment with these HSMs (Fig. 4d) and also, BFU-E colonies were higher in number for 2-APB and TAME treatments (Fig. 4e). These functional CFU assay findings showed that 2-APB, BML-260 and TAME compounds contributed to the multi-lineage potential of expanded UCB cells and maintained the self-renewal and expansion ability of HSM treated UCB-HSCs.

\subsection{BML-260 and 2-APB increased the ALDH ${ }^{\text {hi }}$ enriched mPB cell population}

HSC characterization is mainly performed with immunophenotyping by using surface markers as mentioned above. Another identification method is based on HSC metabolism property. ALDH enzyme activity is a metabolic marker for HSCs because of their glycolysis pathway preference and therefore, HSCs are enriched in 
ALDH ${ }^{\text {hi }}$ population(18). To evaluate the effects of selected HSMs on metabolic profile, we performed the ALDH measurement assay on mPB-MNCs post 7 days of treatment with the effective doses of 2-APB, BML-260 and TAME molecules. We found that treatments of 2-APB and BML-260 treatments significantly increased the $\mathrm{ALDH}^{\text {hi }}$ population ratio approximately 2-fold compared to DMSO control. Besides, TAME compound did not any significant effect on $\mathrm{ALDH} \mathrm{Hr}^{\mathrm{Br}}$ enriched cell population of the expanded mPB cells (Fig. 5a).

Another metabolic effect can be observed on the production of reactive oxygen species in response to increased metabolic activity in response increased proliferation kinetics(19). To detect the effect of enhanced metabolic activity with enhanced cycling potential on HSM-treated MPB cells, we determined the ROS content on mPBMNCs post 7 days of treatment with the effective doses of 2-APB, BML-260 and TAME molecules. We found that any compounds did not considerably increased in the ROS production compared to DMSO control (Fig. 5b).

\subsection{BML-260 and 2-APB molecules outline a safer profile than TAME in HSC expansion}

Next, we evaluated the viability of expanded mPB-HSPCs with 7-AAD staining following 7 days of treatment with the effective doses of 2-APB, BML-260 and TAME molecules. We found no significant change on the viability of the expanded cells with 2-APB, BML-260 and TAME compounds. Treatment with TAME molecule slightly decreased the viability compared to DMSO control (Fig. 6a).

It is known that HSCs protect themselves against to oxidative and genotoxic stresses through staying in quiescence state. Enhanced metabolic activity can result in oxidative stress by Ros production and thus, occurs DNA damage, senescence and apoptosis(20). We performed a multiplex assay (Figure S3A) consisting of measurement of DNA damage, cell cycle and apoptosis to determine the effects of HSMs on expanded mPBHSPCs post 7 days of treatment with effective doses of p300/CBP Inhibitor VI, 2-APB, BML-260 and TAME molecules. TAME molecule was determined to significantly increase cycling cell percentage 3-fold by BrdU incorporation compared to DMSO control (Fig. 6b). Besides, we showed 2-fold increase of the apoptotic and genotoxic effects of TAME molecule compared to DMSO control (Fig. 6c-e). The results showed that TAME molecule increased the cycling cell ratio but increased cell division activity of TAME accompanies the cycling cells to accumulate DNA damage and thus possible higher rates of apoptosis. On the other hand, TAME did not show any significant apoptotic or necrotic effect on mPB-CD34 + cells post treatment according to Annexin $\mathrm{V}$ apoptosis assay (Fig. 6f). The differences between these findings suggested that TAME treatment induced apoptosis and DNA damage on progenitor cells rather than the expanded CD34 + cells. 2-APB, and BML-260 treatments did not show any significant cytotoxic and genotoxic effects on mPB-HSPCs (Fig. 6c-e). We also found that BML-260 treatment significantly increased the viability of the expanded mPB-CD34 + cells and, treatment of 2-APB molecule did not show in any significant apoptotic or necrotic effect on mPB-CD34 + cells (Fig. 6f). Interestingly, we found that p300/CBP Inhibitor VI molecule significantly decreased the cycling cell ratio despite of its increase in human HSPC content and also it showed cytotoxic and genotoxic effect on the expanded cells (Figure S2B). These results revealed that 2-APB and BML-260 molecules are more advantageous comparing to p300/CBP Inhibitor VI and TAME small molecules in terms of their safety profile ex vivo.

\subsection{BML-260 and TAME may modulate the regulators of cell cycle checkpoint and HDR}


To understand what the effect mechanism of BML-260 and TAME compounds on the expansion of human HSPCs, we analyzed the expressions of cell cycle and quiescence modulator genes (S-phase and CDKIs), BML260 and TAME related genes and HDR-related genes on HSM treated human mPB-HSPCs. Post analysis of CDKI expression following the effective doses of these compounds, we showed the downregulation of p19 (CDKN2D) and p27 (CDKN1B) expressions in BML-260 treated cells. Interestingly, TAME treatment downregulated only p27 expression about 4-fold while it upregulated the expressions of p19, p21 and p57 CDKIs about 2-fold compared to DMSO (Fig. 7a).

Next, we determined the variable gene expression profile when we analyzed the expression of S-phase (MCM2, RPA1 and PCNA) and HDR-related genes. If we categorized the genes according to their function on the cells, we found that treatment of BML-260 had adjuvant effect on DNA replication and thus DNA repair mechanism via 3fold upregulation of PCNA and also, enhanced the transition to S-phase via 2-fold upregulation of MCM2 gene (Fig. 7b). In contrast to that, treatment of TAME compound did not effect on expression level of MCM2 while downregulated PCNA expression around 3-fold. We found that the expression of RAD51, XRCC1 and ERCC1 from HDR-related genes except MSH2 gene were declined in BML-260 treated cells. Intriguingly, MSH2 gene expression was upregulated 2-fold post BML-260 treatment. In contrast to that, TAME compound significantly upregulated RAD51 and XRCC1 genes around 2- and 4.5-fold, respectively while did not effect the expression of MSH2 and ERCC1 genes. Once for all, the expression level of RAD17 which is responsible for cell cycle arrest at G2 phase in response to DNA damage, was decreased 2.5 fold on TAME-treated cells while there was any remarkable changes on the expression post BML-260 treatment (Fig. 7b).

We also determined the changes in BML-260 and TAME related gene expressions. We found that the expression of Cdh1 (TAME modulated gene), which participates in cell cycle regulation through composing APC/C $\mathrm{C}^{\mathrm{Cdh} 1}$ complex $(21,22)$, was highly downregulated in TAME-expanded mPB-HSPC. BML-260 treatment downregulated Cdh1 expression. Besides, Skp2 cell cycle regulator gene that is responsible for $\mathrm{p} 27$ degradation and regulated by $\mathrm{APC} / \mathrm{C}^{\mathrm{Cdh} 1}$ for its proteosomal degradation (23), was found to be upregulated up to 4-fold in TAME-treated cells in parallel with downregulation of APC/C ${ }^{\mathrm{Cdh} 1}$ complex. Interestingly, the expression of Skp2 was upregulated up to 6-fold in BML-260-treated cells. The increase of Skp2 expression with BML-260 was correlated with downregulation of Cdh1 in the cells. On the other hand, we evaluated the mRNA expression level of c-Myc gene because of its relation with JNK pathway. JNK pathway (c-Jun NH2-terminal kinases) activation is promoted by Jsp-1 (Jnk Stimulatory Phosphatase-1) phosphatase which can be inhibited by BML-260 small molecule $(24,25)$. We found that c-Myc was significantly downregulated in BML-260-treated cells (up to 3-fold) as expected. TAME treatment, on the other hand, downregulated the expression of c-Myc (Fig. 7c).

These findings revealed that BML-260 molecule showed better effect on cell division than TAME compound by enhancement of cell cycle regulator. TAME compound showed the more adjuvant effect on DNA repair mechanism than BML-260 compound through enhancement of HDR genes.

Recent studies have reported that activation of JNK pathway have either role in apoptotic or anti-apoptotic functions. In addition, JNK pathway contributes to activation of mitochondrial apoptotic pathway through activation of pro-apoptotic members and inhibition of anti-apoptotic members of the Bcl2-related protein family following phosphorylation of them(26). Therefore, we analyzed apoptosis-related genes (Fig. 7c) since it was known that the major role of JNK pathway in apoptosis. We found that the expression of Bax (Bcl-2 associated x) which acts pro-apoptotic function regulated by p53(27), was downregulated at 2-fold in BML-260 treated cells 
in despite of upregulation of p53 around 2-fold. This downregulation was expected by inhibition of JNK pathway with BML-260 inhibitor. TAME treatment also did not show any significant changes in the expressions of Bax and p53. Intriguingly, TAME treatment resulted in downregulation of Bcl-2 gene expression, which acts antiapoptotic function, at 2-fold. These findings reveal that TAME treatment may inhibit APC/C ${ }^{C d h} 1$ by downregulation of Cdh1 gene and thus, provides cell cycle progression at G1/S transition via degradation of p27 through prevention of degradation of Skp2. Unexpected downregulation of Cdh1 and upregulation of Skp2 on BML-260-treated cells reveal that BML-260 treatment may promote the negatively regulation of $A P C / C^{C d h} 1$ and thus, cell cycle progression. Downregulation of c-Myc could also contribute to HSPC expansion. Besides, the positive effect of BML-260 on cell viability may be related to downregulation of Bax apoptotic gene (Fig. 7c).

\subsection{Repopulation and engraftment abilities of BML-260 and TAME expanded HSCs}

Reconstitution of hematopoietic system is a main function of transplanted HSCs for hematopoietic recovery and also, a major handicap for HSCT. Next, we performed the functional assay to detect the in vivo efficacy of the expanded HSCs in the reconstitution of hematopoiesis. We evaluated the repopulation abilities of BML-260 treated HSCs with their short-term (at 4 weeks) and long-term (at 20 weeks) multilineage engraftment following the xenotransplantation of human PB-CD34 + cells to SCID mice, also known as immunocompromised mice resulting lack of $\mathrm{T}$ and $\mathrm{B}$ cells (Fig. 8a). For that, we collected the peripheral blood from recipient mice through retro-orbital bleeding. We indicated the repopulation and multilineage engraftment in the presence of hCD $45+$ $\mathrm{CD} 33+$ myeloid cells, $\mathrm{hCD} 45+\mathrm{CD} 3+\mathrm{T}$ and $\mathrm{hCD} 45+\mathrm{CD} 19+\mathrm{B}$ lymphoid cells in the peripheral blood according to the modified protocol(28). We found that BML-260 expanded HSCs repopulated the T lymphocytes, B lymphocytes and myeloid cells in the long-term engraftment (Fig. 8b-c). We also analyzed the human HSPC contents in recipient BM and able to show human HSPC content with transplantation of BML-260 expanded HSCs (Fig. 8d). Quantification of hCD45 + cell percentage showed the enhanced engraftment ability of expanded HSCs in long-term compared to short-term (Fig. 8e). These findings suggested that BML-260 expanded HSCs functionally reconstituted the entire hematopoiesis in LT-engraftment.

\subsection{Development of HSM cocktail for human HSPC Expansion}

Cellular processes are carried out by collaboration of many pathways in a cell. HSCs have dynamic structure because of either their niche regulation or their role in hematopoiesis. Enhancement of their cell division capacity can be required for modification of various collaborative pathways at once. Herein, the development of combinational HSM cocktain, which could regulate multiple pathways at the same time in the cells, was sought for human HSC expansion. From this point of view, we focused on the development of HSM cocktail for human UCB-HSC expansion with our HSM pool. For that, we prepared different HSM mixtures with all $35 \mathrm{HSM}$. Strategy of mixture preparation using in this study was schematically presented in Fig. 9a. We prepared 36 mixtures at three different working concentrations and each mixture included the all HSMs except one of them respectively, while the one mixture included all of them. Therefore, we determined the effect of each HSMs by this way for elimination of ineffective or nonessential HSMs. We analyzed the HSPC content post 7 days of treatment with these mixtures. We compared the results to DMSO and ALL mixture including all HSMs controls. We found that

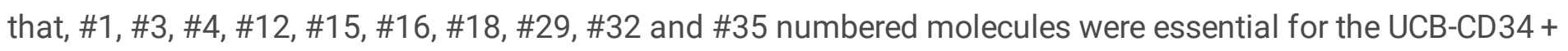
CD38- cell expansion. On the light of these results, 13 HSMs including \#1, \#3, \#4, \#11, \#12, \#18, \#22, \#23, \#25, $\# 26$, \#29, \#32 and \#35, was selected for further study (Fig. 9b-c-d). For the second elimination, the new mixtures 
were prepared by the same way as described before. When the results were compared to results of ALL(13HSM) mixture, we found that \#1, \#22, \#23,\#26, \#32 and \#35 numbered molecules were found to be essential for CD34 + cell expansion and were indispensable for CD34 + CD38- cell expansion for at $0.01 \mu \mathrm{M}$ dose/each. Given these findings, we decided utilization of \#1, \#11, \#22, \#23, \#26, \#32 and \#35 molecules to prepare the new mixtures (Fig. 9e-f). We also prepared the new mixture with these selected 7 HSMs and the treated cells were analyzed post 7 days of treatment about HSC content. We found that any concentration or combination of the new mixture did not improved CD34 + and CD34 + CD38- cell expansion compared to DMSO in a dose dependent manner (not shown). Taken together, these findings suggest that there should further addition of potent small molecules to HSM pool and perform additional studies to achieve establishment of a HSM cocktail for human HSC expansion, which is superior than BML-260 treatment.

\subsection{Chemically-defined HSC medium supplemented with BML- 260 molecule improves HSC expansion in culture}

Development of HSC expansion medium including the specific agents that modulate the HSC function is point of focus for stem cell researchers especially for GMP compatible platforms. To develop human HSC expansion medium with enhanced performance, we compared two basal medium types RPMI 1640 and IMDM prepared with different serum concentrations and ITS supplement, which is known to be used for serum-free medium to reduce the serum amount. Besides, BML-260 which was found to be more potent than TAME molecule, was used for small molecule supplement and also, cytokine combinations (SCF, TPO and FLT3) were used for every medium compositions (Fig. 10a). Post 7 days culture of mPB-HSPCs in these medium compositions, human HSPC content analysis was done. IMDM composition supplemented with 5\% FBS, cytokines and BML-260 molecule increased the frequency of CD34 + CD38- cell population (Fig. 10b-c). In addition, we tested that RPMI 1640 compositions. The most effective medium composition was identified as RPMI 1640 supplemented with $10 \%$ FBS, cytokine combinations, ITS and BML-260. This medium composition increased the CD34 + cell frequency around 2-fold and CD34 + CD38- cell frequency around 2.5-fold (Fig. 10d-e). These results suggest that this medium combination could be ideal for a development of chemically-defined human HSC expansion medium.

\section{Discussion}

Reconstitution of hematopoietic system have critical importance in clinical medicine. Many people in the worldwide are suffered from hematological malignancies and disorders, autoimmune disorders and also, genetic blood-related diseases $(4,29)$. Most promising approach of treatment of these diseases is HSCT. Despite advantages of UCB source including no requirement of surgical intervention and HLA matching, and also reduced incidence of GVHD, reduced hematopoietic recovery due to lack of sufficient HSC number, is a major problem for UCB-HSCT.

Main criteria of ex vivo expansion of HSCs is based on symmetric cell division, enhanced self-renewal capacity without differentiation and over-cycling(30). These handicap and criteria have been considered for the development of ex vitro HSC expansion technologies. In addition, utilization of cytokines/growth factors, molecular regulator pathway components and co-culture with neighbor cells involved in their microenvironment have been initial approaches. Several culture systems containing the previously identified factors have been developed as 2D and 3D or liquid and perfusion systems(31-33). 
To this end, we screened small molecule pool containing 35 different compounds. Our strategy on creation of hematopoietic small molecule pool was based on analysis of role of different genes on HSC function and then, their protein inhibitors were selected depending on their druggable property and also, availability of small molecules. We hypothesized that targeting to HSC quiescence factors by putative HSMs could be induce HSC expansion ex vivo and in vivo. Therefore, we aimed to identify the most effective HSMs and HSM combinations to develop chemically defined human HSC expansion medium. For this aim, we used two HSC sources; mobilized peripheral blood and umbilical cord blood for the comparison.

Small molecules affect many pathway components involved in regulation of self-renewal, proliferation, differentiation, apoptosis, adhesion, migration and also interaction with other cells on HSCs. In presence of these molecules cause either upregulation of target pathways or downregulation of proteins(6). Taken together, SMs could be therapeutically used for ex vitro and in vitro HSC expansion. We hypothesized that targeting to HSC quiescence factors by putative HSMs could be induce HSC expansion ex vivo and in vivo. We aimed to identify the most effective HSMs and HSM combinations to develop chemically-defined human HSC expand medium. Treatments with 2-APB, BML-260, TAME and MEIS1 inhibitors among all HSMs highly increased the CD34+ and ALDH ${ }^{\text {hi }}$ cell populations of UCB-HSPC content compared to DMSO control. Treatments with B ML-260 and TAME compounds also increased UCB total cell count. In similar with UCB cells, p300/CBP inhibitor VI had a highly adjuvant effect on $\mathrm{m}$ PB CD34+. CBP is required for self-renewal while $\mathrm{p} 300$ is needed for differentiation in hematopoiesis(34). Potentially inhibitor effect of P300/CBP inhibitor VI on p300 component comparing to CBP can explain the positive regulation of p300-CBP inhibitors on human HSPC pool. However, we found that the inhibitor VI has highly apoptotic and genotoxic effect on $\mathrm{m}$ PB HSPCs.

Another molecule that enhanced the human HSPC content is 2-Aminoethoxydiphenyl borate (2-APB) identified as an antogonist of inositol 1,4,5-trisphosphate (InsP3R). This molecule inhibits InsP3-induced $\mathrm{Ca}+2$ release from mouse cerebellar membranes in a concentration-dependent manner and also, found as a blocker of storeoperated $\mathrm{Ca}+2$ entry (SOCE) independently of InsP3 $(35,36)$. Moreover, it promotes the gradual release of $\mathrm{Ca} 2+$ stores through inhibiting sarco-endoplasmic reticulum Ca2+ ATPases (SERCAs) pumps. Calcium metabolism involved in HSC niche regulates HSC maintenance, quiescence and self-renewal(12). Endosteal niche where HSC localize in $\mathrm{BM}$ is characterized by high calcium concentrations found in osteoclasts in the niche. HSCs highly express calcium sensing receptors (CaRs) which is a $\mathrm{G}$ protein-coupled receptor to provide sensitivity against to calcium abundance in response to niche. The effect of CaRs on HSC lodgement mainly regulates HSC homing(37).

2-APB is an antogonist of inositol 1,4,5-trisphosphate (InsP3R) This molecule inhibits InsP3-induced Ca+2 release from mouse cerebellar membranes(35). HSCs highly express calcium sensing receptors (CaRs) which is a $\mathrm{G}$ protein-coupled receptor to provide sensitivity against to calcium abundance in response to niche(37). 2APB treatment allows ex vitro expansion of UCB-HSC through triggering re-entry to S/G2/M phase during cell division. Any alterations were not detected on cycling ability of $\mathrm{m}$ PB-CD34+cells in despite of the increase in mPB-HSPC content. Findings reveal that 2-APB molecule is more promising for ex vivo expansion of UCB- HSC rather than $\mathrm{MPB}$ hematopoietic cells.

Next, we focused on the BML-260 and TAME molecules that regulated the both UCB- and m PB-HSC expansion. BML-260 small molecule is an inhibitor of dual specificity phosphatase JSP-1 (DUSP22). Jsp-1 promotes activation of JNK pathway which is an evolutionarily conserved subclass of mitogen-activated protein (MAP)

Page $14 / 28$ 
kinases and thus, is involved in signal transduction(24,25). Besides, TGF- $\beta$ signaling which regulate HSC quiescence and inhibits HSPC proliferation can be promoted through JNK pathway\{Hinge, 2016 \#35\}.

The role of JNK pathway in hematopoiesis makes think that how targeting of JNK pathway affect on HSC expansion. Xiong Xiao et.al showed that JNK inhibitor, JNK-IN-8 highly induced in vitro expansion of CD34+CD45RA-, and CD34+CD38-CD45RA-CD90+ of UCB and increased in vivo long-term repopulation capacity of expanded cells through downregulation of c-Jun gene(38). Here, we used Jsp-1 inhibitor BML-260 to expand human mPB and UCB HSC. We found that BML-260 molecule induced both mPB- and UCB-derived HSPC expansion in culture. On the other hand, we analzyed whether this molecule modulates cell cycle profile of expanded cells and we found that BML-260 increased the frequency of mPB-CD34+ cell at G1 phase and the frequency of UCB-CD34+ at S/G2/M phase (up to 5 fold) through decrease in the frequency of quiescent cells (at $\mathrm{GO}$ phase). Besides, we detected slight increased proliferation rate on PB-CD34+ cells with CFSE proliferation assay. Moreover, BML-260 increased multi-lineage potential of UCB-HSPCs with their role in cycling potential. BML-260 molecules upregulated the expression of MCM2 and PCNA, DNA replication-related genes on m PBHSPCs. Downregulation of p27 is dependent on upregulation of Skp2 in B ML-260-treated cells. Cdh1 is tightly regulated by JNK pathway(39) and inactivation of Cdh1 is related to enhancement of SkP2 function(40) and thus, degradation of p27. BML-260 molecule did not show any genotoxic or apoptotic effect on expanded cells. We also showed increased viability on BML-260 treated cells in parallel with decreased apoptosis. Xenotransplantation study showed that BML-260 expanded HSCs displayed enhanced multilineage reconstitution capacity in the long-term engraftment. Besides, transplanted HSC's also triggered the murine hematopoiesis. These findings reveal that Bml-260 induces ex vivo expansion of human mPB and UCB derived HSPC through modulation of cycling potential.

Tosyl-I-arginine methyl ester (TAME) is an inhibitor of anaphase-promoting complex/cyclosome (APC/C) TAME molecule inhibits the APC activation through substitution of the isoleucine-Arginine (IR) tail-binding sites of Cdc20 and Cdh1 via its structurally similarity with IR tail of coactivators(22). The switching between APC/CCDC20 and APC /CCdh1 cooperates cell cycle progression(21). The function of APC with Cdh 1/Cdc20 coactivator on cell cycle regulation differs between cycling and quiescent cells. In hematopoietic cells, it was reported that downregulation of Cdh1 which is known to be highly expressed in human CD34+ cells, by its inhibitor Emi1 resulted in promote re-entry to cell cycle and proliferation of HSCs(41). Taken together, we focused on TAME inhibitor of APC/C complex and we found that TAME treatment induced the both UCB- and mPB-HSC expansion.

According to gene expression analysis, we found that the expression of p27 CDKI was downregulated in mPBHSPCs with inhibition of APC-mediated proteolysis. Skp2 expression was upregulated in TAME-treated cells in addition to downregulation of Cdh1 gene. TAME inhibitor may provide HSC proliferation through increased Skp2 function-dependent p27 degradation with inhibition of Cdh1 activation. TAME treatment showed slight increasement of ROS level in the expanded $\mathrm{m}$ PB cells. TAME treatment also displayed apoptotic and genotoxic effect on the expanded cells. The apoptotic effect of TAME molecule may be dependent on p57-mediated apoptosis. Besides, anti-apoptotic gene Bcl-2 was downregulated in TAME-treated cells. These findings reveal that TAME molecule increased human mPB and UCB HSPC expansion through notably inhibition of Cdh1 and thus, modulation of cell cycle kinetics, but its side effects on expanded cells are remarkable point. 
On the light of these all findings, we determined that BML-260 and TAME molecules are promising for both human mPB- and UCB-derived HSC ex vivo expansion. BML-260 and TAME contribute to human HSC expansion through modulation of cell cycle kinetics. BML-260 molecule is more potent to expand human HSC with no cytotoxic and genotoxic effect. Enhanced repopulation ability of BML-260 treatment confirmed its utilization for human ex vivo HSC expansion. On the other hand, the chemically-defined HSC medium composition including RPMI 1640 basal medium supplemented with cytokine combinations (SCF, TPO and FLT-3), 10\% FBS, ITS and BML-260 molecule could be promising for development of chemically-defined human HSC expansion medium post detail investigations and validations.

\section{Declarations}

Funding and acknowledgments: We thank the support from the European Commission Co-Funded Brain Circulation Scheme by The Marie Curie Action COFUND of the 7th Framework Programme (FP7) (115C039). EA had been supported by TÜBITAK-BIDEB 2211 program. We would like to thank to Prof.Dr. Zafer Gülbaş, Prof. Dr. Rukset Attar, Opr. Dr. Elif Ganime, Dr. Neslihan Meriç, Emre Can Tüysüz, Utku Özbey for their support in supply of the human samples and FACS analysis.

Authors' information: EA planned the experiments, collected and analyzed data, and wrote the manuscript. RDT and SA contributed to xenotransplantation study. FK designed the studies and wrote the manuscript.

Availability of data and material. Additional supporting data is available in the supplementary files. Further information is available upon request.

Consent for publication: All authors are read and approved manuscript for publication.

Ethics approval and consent to participate: UCB samples were obtained from newborns with their parent's permission by Yeditepe University Hospital. mPB samples were obtained from healthy individuals with their permission by Anatolian Health Center. The SCID mice were used for in vivo studies. All human and animal studies were approved by the Institutional Clinical Studies Ethical Committee of Yeditepe University (Decision numbers 1067 and 944), and the Institutional Animal Care and Use Committee of Yeditepe University (YUDHEK, decision number 429). All human and animal studies were approved by the Institutional Clinical Studies Ethical Committee of Yeditepe University (Decision numbers 1067 and 944), and the Institutional Animal Care and Use Committee of Yeditepe University (YUDHEK, decision number 429).

Conflict of interest: FK is founder of Meinox (www.meinoxtech.com). All other authors declare no conflict of interest.

\section{References}

1. Orkin SH, Zon LI. Hematopoiesis: An Evolving Paradigm for Stem Cell Biology. Cell. 2008;132(4):631-44.

2. Hofmeister CC, Zhang J, Knight KL, Le P, Stiff PJ. Ex vivo expansion of umbilical cord blood stem cells for transplantation: Growing knowledge from the hematopoietic niche. Bone Marrow Transplant. 2007;39(1):11-23.

3. Park SK, Won JH. Usefulness of umbilical cord blood cells in era of hematopoiesis research. Int J Stem Cells. 2009; 
4. Kelly SS, Sola CBS, de Lima M, Shpall E. Ex vivo expansion of cord blood. Bone Marrow Transplant [Internet]. 2009;44(10):673-81. Available from:

http://www.ncbi.nlm.nih.gov/pubmed/19802023\%5Cnhttp://www.pubmedcentral.nih.gov/articlerender.fcgi? artid=PMC4157906

5. Shpall EJ, Quinones R, Giller R, Zeng C, Baron AE, Jones RB, et al. Transplantation of ex vivo expanded cord blood. Biol Blood Marrow Transplant. 2002;

6. Zhang Y, Gao Y. Novel chemical attempts at ex vivo hematopoietic stem cell expansion. Int J Hematol. 2016;103(5):519-29.

7. Feng Y, Xie XY, Yang YQ, Sun YT, Ma WH, Zhou PJ, et al. Synthesis and evaluation of pyrimidoindole analogs in umbilical cord blood ex vivo expansion. Eur J Med Chem. 2019;

8. Kumar S, Geiger H. HSC Niche Biology and HSC Expansion Ex Vivo. Trends in Molecular Medicine. 2017.

9. Tarunina M, Hernandez D, Kronsteiner-Dobramysl B, Pratt P, Watson T, Hua P, et al. A Novel High-Throughput Screening Platform Reveals an Optimized Cytokine Formulation for Human Hematopoietic Progenitor Cell Expansion. Stem Cells Dev. 2016;

10. Yao CL, Chu IM, Hsieh TB, Hwang SM. A systematic strategy to optimize ex vivo expansion medium for human hematopoietic stem cells derived from umbilical cord blood mononuclear cells. Exp Hematol. 2004;

11. Health MOF, Medicines T, Agency MD. Republic of Turkey Ministry of Health Turkish Medicines and Medical Devices Agency Good Manufacturing Practices ( Gmp ) Guide for Manufacturing Plants of. 2018;2018:1237.

12. Uslu M, Albayrak E, Kocabaş F. Temporal modulation of calcium sensing in hematopoietic stem cells is crucial for proper stem cell expansion and engraftment. J Cell Physiol. 2020;

13. Kocabas F. Development of Small Molecule MEIS Inhibitors that modulate HSC activity Raife. 2020;1-9. Available from: https://www.biorxiv.org/content/10.1101/2020.02.12.946491v1

14. Kocabas F, Xie L, Xie J, Yu Z, DeBerardinis RJ, Kimura W, et al. Hypoxic metabolism in human hematopoietic stem cells. Cell Biosci. 2015;

15. Aksoz M, Albayrak E, Aslan GS, Turan RD, Alyazici LY, Siyah P, et al. c-Myc Inhibitor 10074-G5 Induces Murine and Human Hematopoietic Stem and Progenitor Cell Expansion and HDR Modulator Rad51 Expression. Curr Cancer Drug Targets. 2018;

16. Simsek T, Kocabas F, Zheng J, Deberardinis RJ, Mahmoud Al, Olson EN, et al. The distinct metabolic profile of hematopoietic stem cells reflects their location in a hypoxic niche. Cell Stem Cell. 2010;

17. Yucel D, Kocabas F. Developments in hematopoietic stem cell expansion and gene editing technologies. In: Advances in Experimental Medicine and Biology. 2018.

18. Hess DA, Meyerrose TE, Wirthlin L, Craft TP, Herrbrich PE, Creer MH, et al. Functional characterization of highly purified human hematopoietic repopulating cells isolated according to aldehyde dehydrogenase activity. Blood. 2004;

19. Ito K, Ito K. Hematopoietic stem cell fate through metabolic control. Exp Hematol. 2018;

20. Shao L, Li H, Pazhanisamy SK, Meng A, Wang Y, Zhou D. Reactive oxygen species and hematopoietic stem cell senescence. In: International Journal of Hematology. 2011.

21. Marashiyan M, Kalhor H, Ganji M, Rahimi H. Effects of tosyl-L-arginine methyl ester (TAME) on the APC/c subunits: An in silico investigation for inhibiting cell cycle. J Mol Graph Model. 2020; 
22. Skaar JR, Pagano M. Control of cell growth by the SCF and APC/C ubiquitin ligases. Current Opinion in Cell Biology. 2009.

23. Hu D, Qiao X, Wu G, Wan Y. The emerging role of APC/CCdh1 in development. Seminars in Cell and Developmental Biology. 2011.

24. Cutshall NS, O’Day C, Prezhdo M. Rhodanine derivatives as inhibitors of JSP-1. Bioorganic Med Chem Lett. 2005;

25. Weston CR, Davis RJ. The JNK signal transduction pathway. Current Opinion in Cell Biology. 2007.

26. Putcha G V., Le S, Frank S, Besirli CG, Clark K, Chu B, et al. JNK-mediated BIM phosphorylation potentiates BAX-dependent apoptosis. Neuron. 2003;

27. Lin A. Activation of the JNK signaling pathway: Breaking the brake on apoptosis. BioEssays. 2003.

28. Park CY, Majeti R, Weissman IL. In vivo evaluation of human hematopoiesis through xenotransplantation of purified hematopoietic stem cells from umbilical cord blood. Nat Protoc. 2008;

29. Hügle T, Daikeler T. Stem cell transplantation for autoimmune diseases. Haematologica. 2010.

30. Tajer P, Pike-Overzet K, Arias S, Havenga M, Staal F. Ex Vivo Expansion of Hematopoietic Stem Cells for Therapeutic Purposes: Lessons from Development and the Niche. Cells. 2019;

31. Tung SS, Parmar S, Robinson SN, De Lima M, Shpall EJ. Ex vivo expansion of umbilical cord blood for transplantation. Best Pract Res Clin Haematol [Internet]. 2010;23(2):245-57. Available from: http://dx.doi.org/10.1016/j.beha.2010.06.004

32. Sorrentino BP. Clinical strategies for expansion of haematopoietic stem cells. Nat Rev Immunol. 2004;4(11):878-88.

33. Aggarwal R, Lu J, Pompili VJ, Das H. Hematopoietic stem cells: transcriptional regulation, ex vivo expansion and clinical application. Curr Mol Med [Internet]. 2012;12(1):34-49. Available from:

http://www.ncbi.nlm.nih.gov/pubmed/22082480\%5Cnhttp://www.pubmedcentral.nih.gov/articlerender.fcgi? artid=PMC3286491

34. Rebel VI, Kung AL, Tanner EA, Yang H, Bronson RT, Livingston DM. Distinct roles for CREB-binding protein and p300 in hematopoietic stem cell self-renewal. Proc Natl Acad Sci U S A. 2002;

35. Peppiatt CM, Collins TJ, Mackenzie L, Conway SJ, Holmes AB, Bootman MD, et al. 2-Aminoethoxydiphenyl borate (2-APB) antagonises inositol 1,4,5-trisphosphate-induced calcium release, inhibits calcium pumps and has a use-dependent and slowly reversible action on store-operated calcium entry channels. Cell Calcium. 2003;

36. Bootman MD, Collins TJ, Mackenzie L, Roderick HL, Berridge MJ, Peppiatt CM. 2-Aminoethoxydiphenyl borate (2-APB) is a reliable blocker of store-operated Ca 2+ entry but an inconsistent inhibitor of InsP 3 -induced Ca 2+ release. FASEB J. 2002;

37. Lam BS, Cunningham C, Adams GB. Pharmacologic modulation of the calcium-sensing receptor enhances hematopoietic stem cell lodgment in the adult bone marrow. Blood. 2011;

38. Xiao X, Lai W, Xie H, Liu Y, Guo W, Liu Y, et al. Targeting JNK pathway promotes human hematopoietic stem cell expansion. Cell Discov. 2019;

39. Gutierrez GJ, Tsuji T, Chen M, Jiang W, Ronai ZA. Interplay between Cdh1 and JNK activity during the cell cycle. Nat Cell Biol. 2010; 
40. Bashir T, Dorello HV, Amador V, Guardavaccaro D, Pagano M. Control of the SCFSkp2-Cks1 ubiquitin ligase by the APC/C Cdh1 ubiquitin ligase. Nature. 2004;

41. Ewerth D, Schmidts A, Kuegelgen B, Wider D, Schüler J, Engelhardt M, et al. Dissecting Stem Cell Proliferation and Differentiation In Association With The Central Cell Cycle Regulator APC/CCdh1 In Vitro and In Vivo.

Blood. 2013;

\section{Figures}

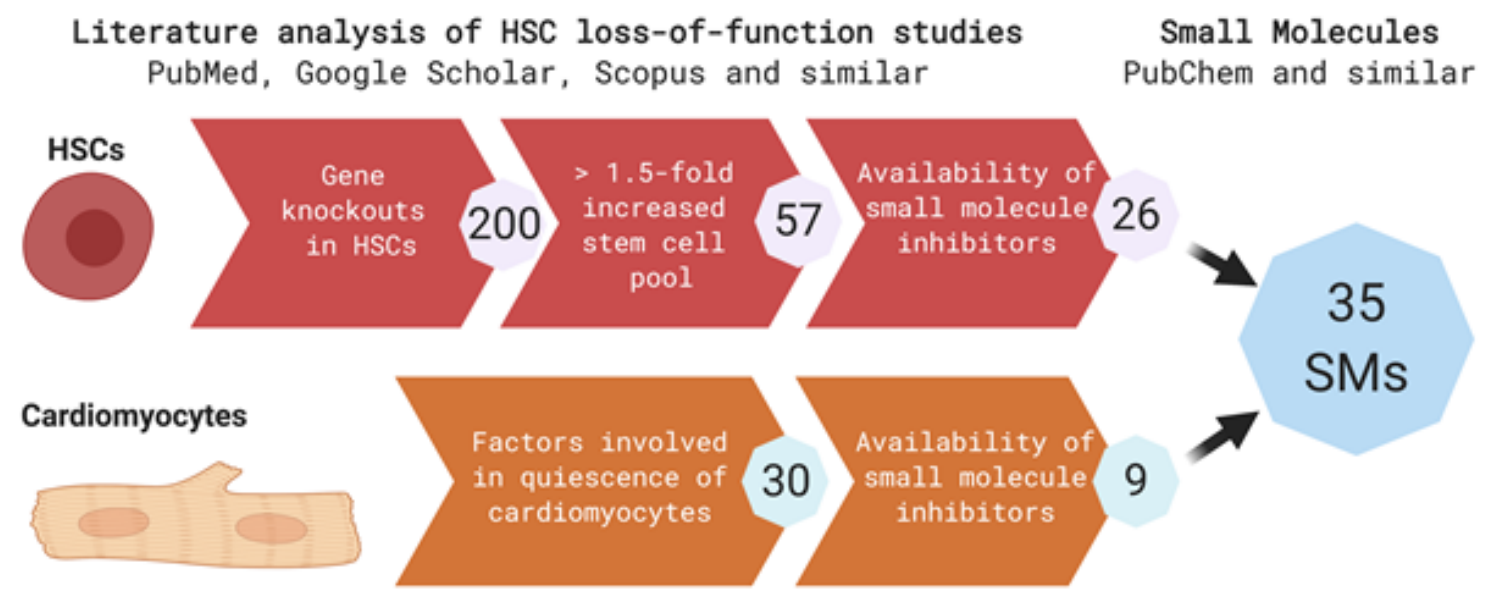

\section{Figure 1}

Identification of putative hematopoietic small molecules (HSMs) that could be effective in human HSC expansion.

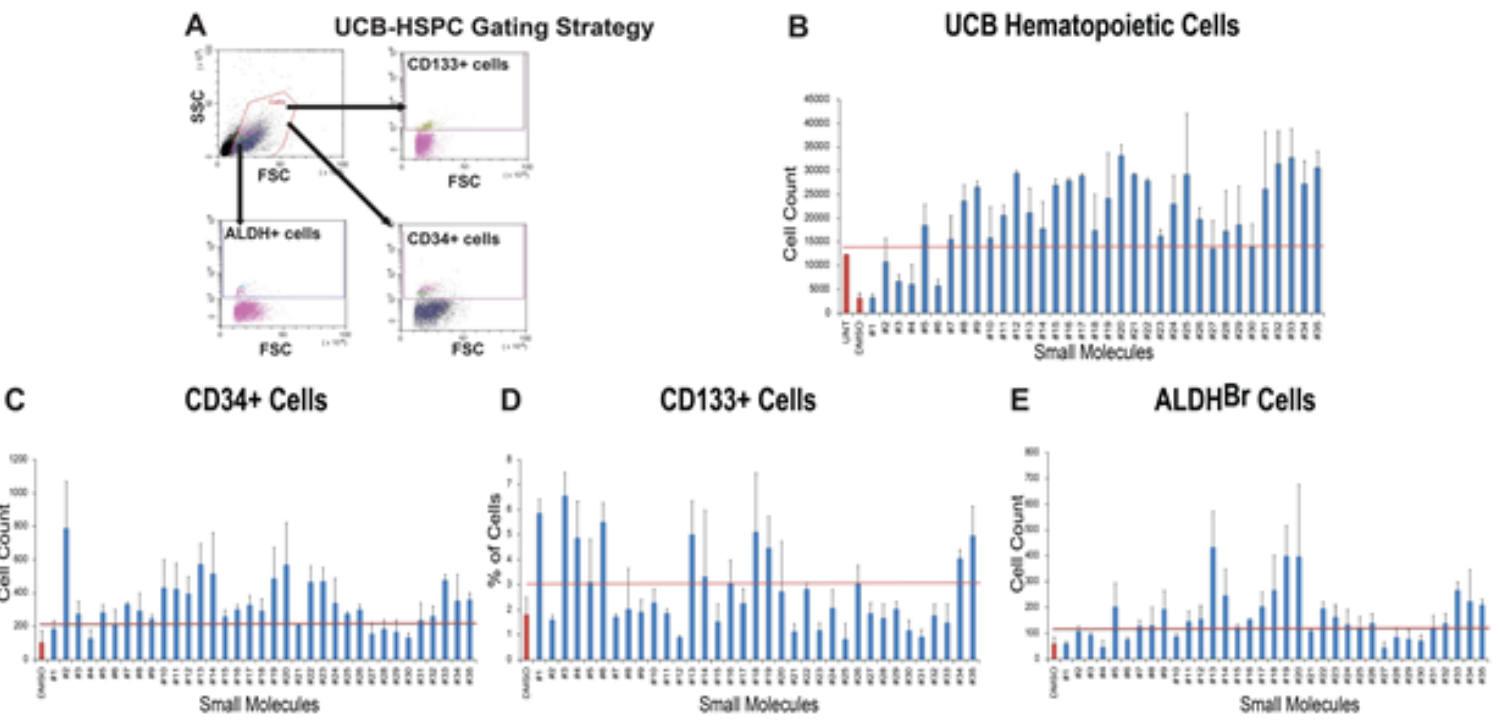

B UCB Hematopoietic Cells

Figure 2 
Screening of human UCB-HSPC content post putative HSM treatments. (a) Gating strategy of flow cytometry analysis for determination HSC content was presented. Quantification of (b) total cell count, (c) CD34+, (d) CD133+ and (e) ALDHhi cell content of UCB-HSPCs were shown as the average of three doses compared to DMSO control $(n=3)$.
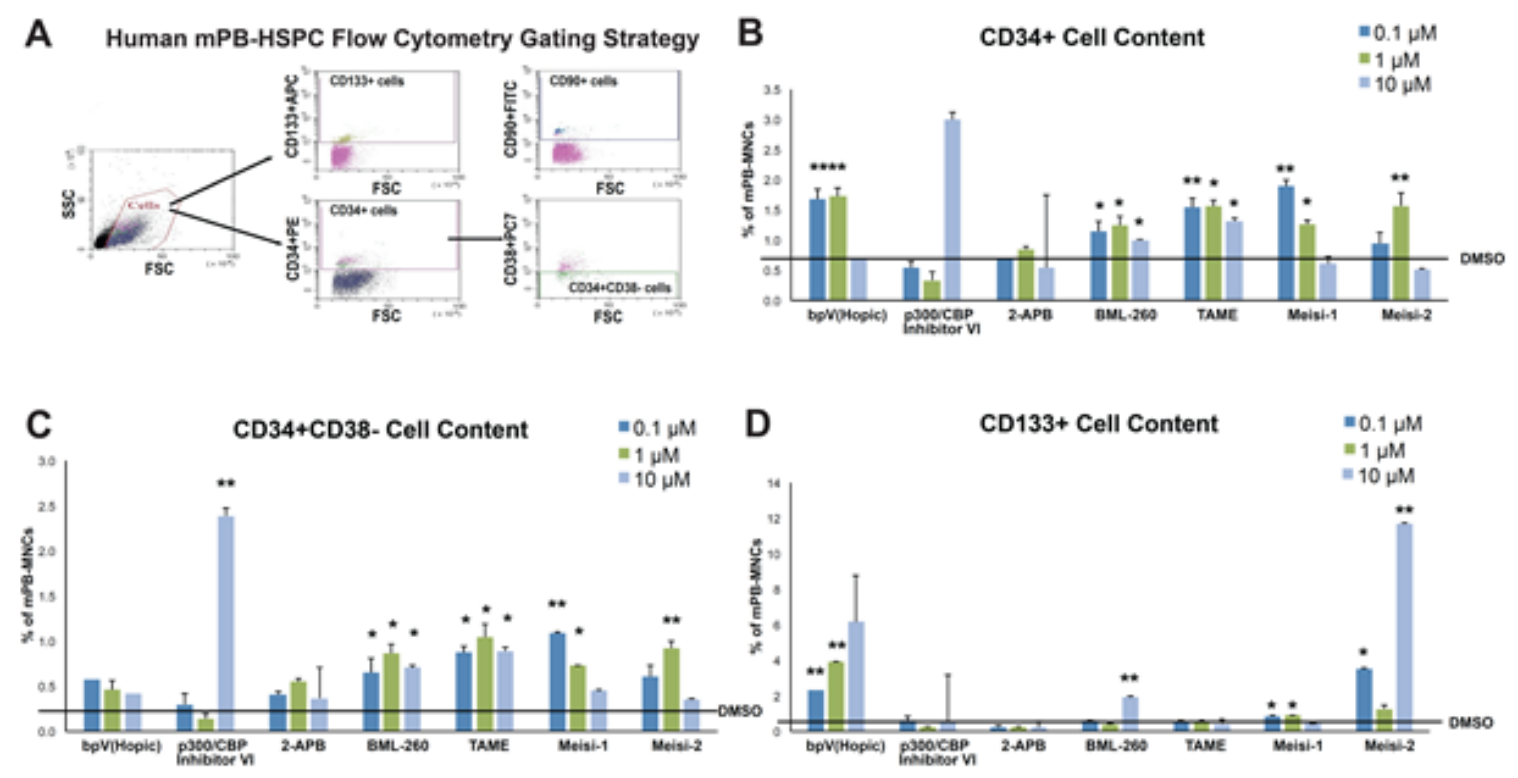

\section{Figure 3}

Analysis of human mPB-HSPC content post treatment of selected HSMs. (a) Gating strategy of human mPBHSPCs analysis. Dose dependent effects of the prominent HSMs on mPB-HSPCs were shown for (b) CD34+ cell content, (c) CD34+CD38- cell content and (d) CD133+ cell content compared to DMSO control. $(n=3 ; * p<0,05$, $\left.{ }^{*} \mathrm{p}<0,01\right)$. 

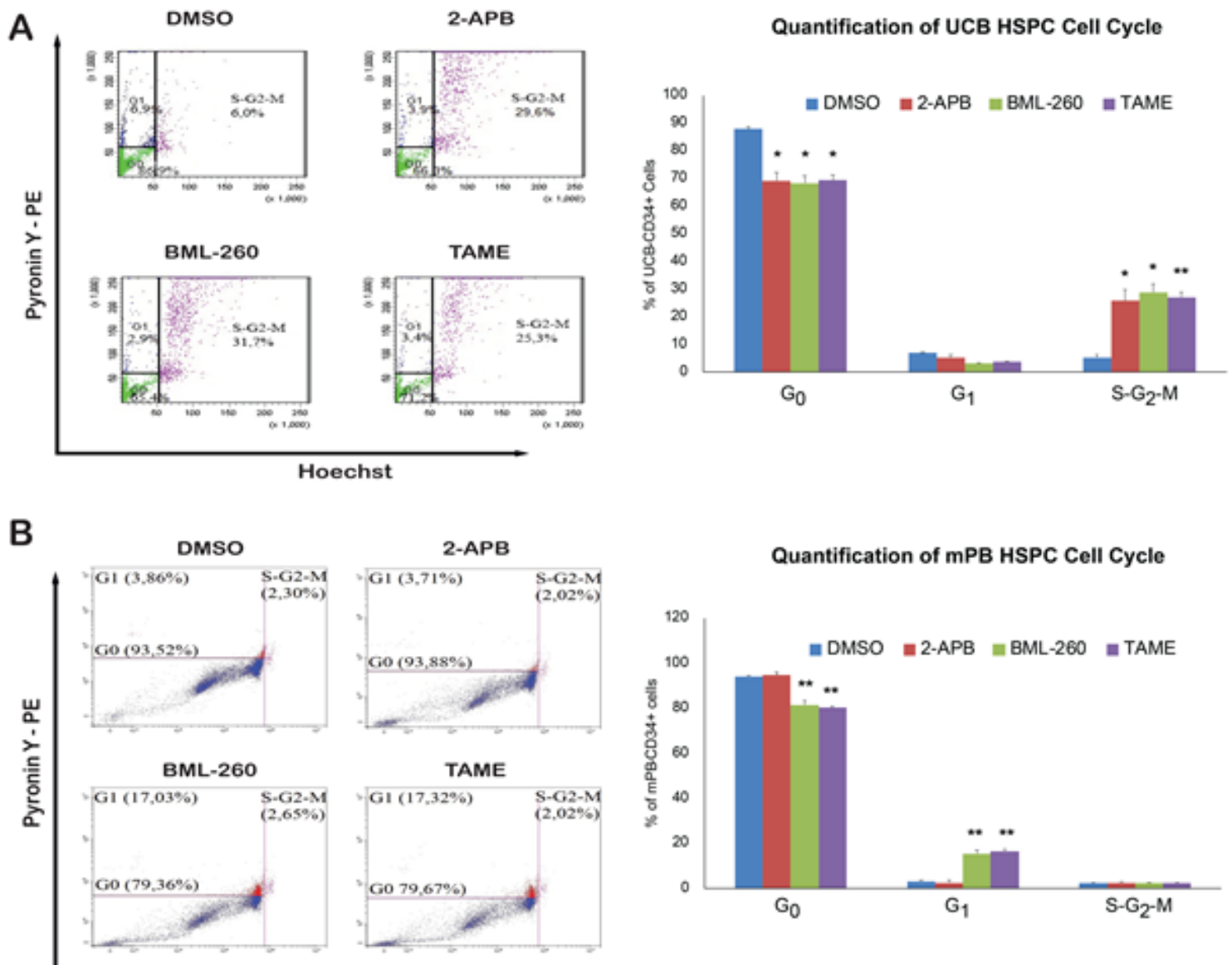

Hoechst
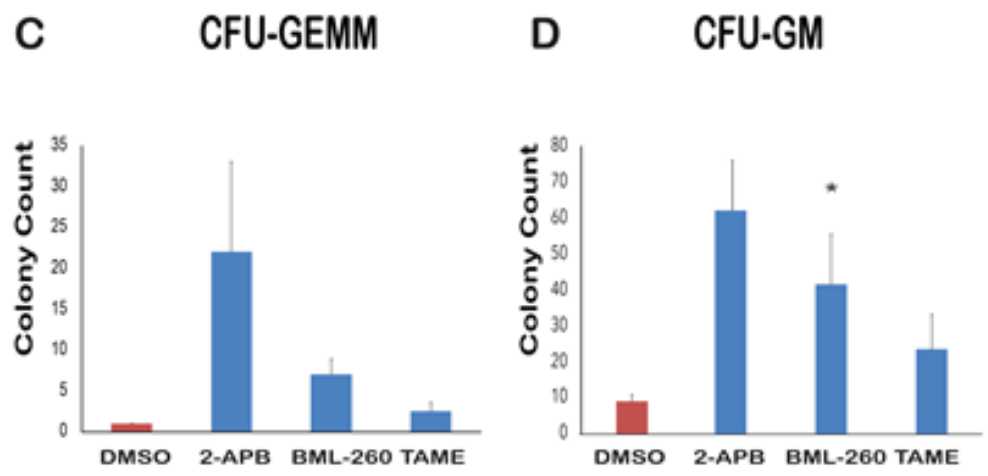

E BFU-E

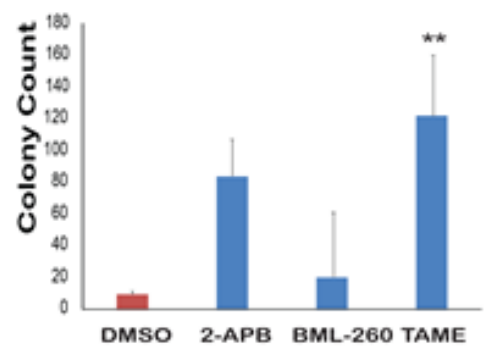

\section{Figure 4}

The effects of prominent HSMs on cell cycle kinetics and multilineage potential of human HSPCs. Cell cycle analysis of HSM expanded-UCB- (a) and mPB-CD34+ cells (b) post 4 days of treatment were shown. Left: Representative flow plots. Right: Quantifications compared to DMSO control. HSM expanded-UCB-HSPCs were quantified for CFU-GEMM (c), CFU-GM (d) and BFU-E (e) colony types compared to DMSO control. $\left(n=3\right.$; ${ }^{*} p<0,05$, $\left.{ }^{\star *} \mathrm{p}<0,01\right)$. 


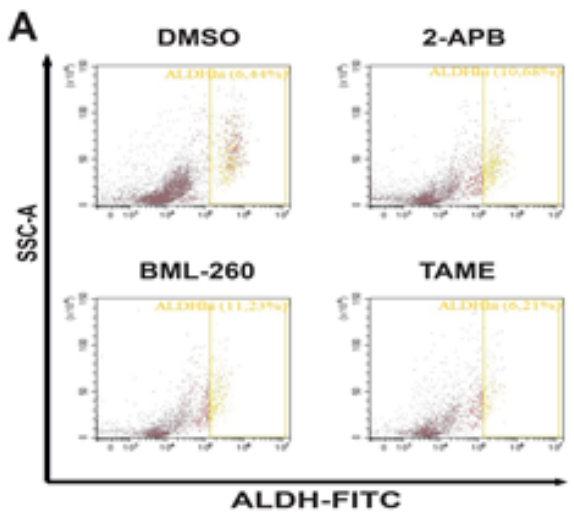

Quantification of ALDHBr Content
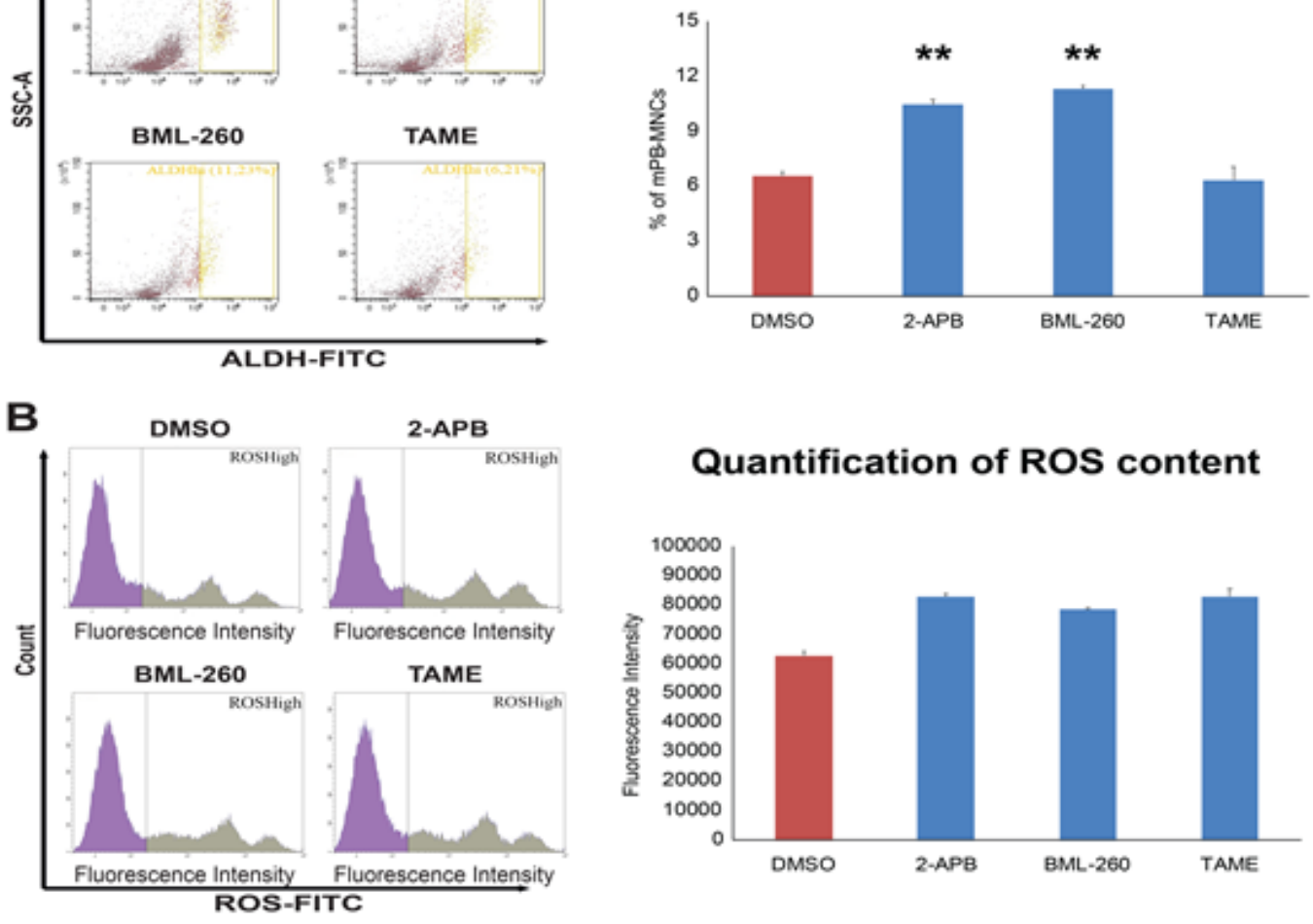

\section{Quantification of ROS content}

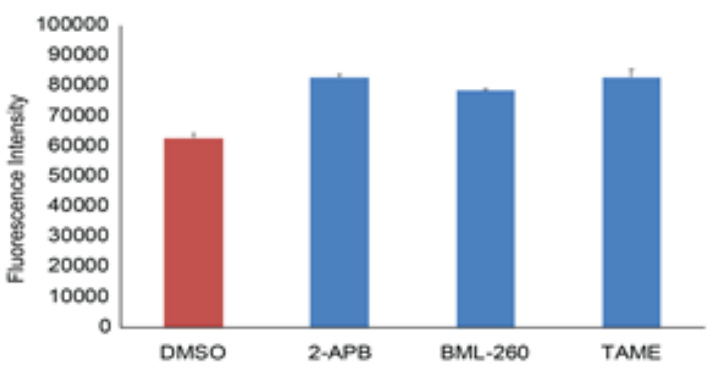

Figure 5

The effects of prominent HSMs on metabolic profile of mPB-HSPCs post 7 days of treatment. (a) The ALDHhi cell content post treatment in comparison to DMSO. (b) ROSHigh content of HSM expanded-cells. Left: Representative ROS flow plots. Right: Quantification of ROS levels result compared to DMSO control. ( $n=3$; $\star * p<0,01)$. 
A

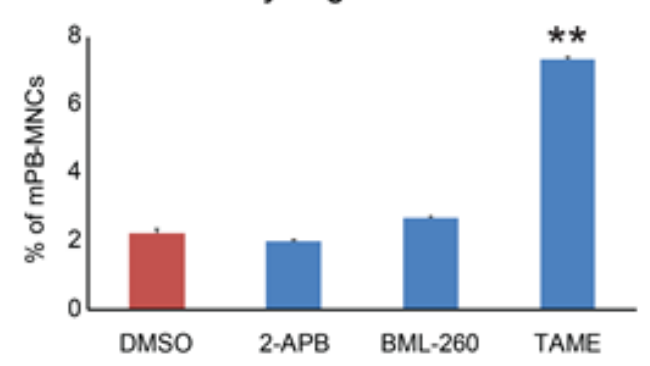

C

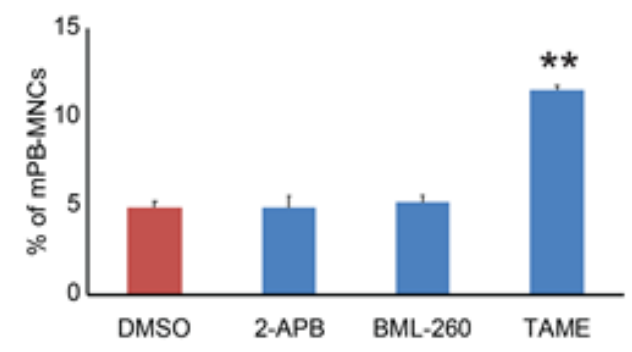

E

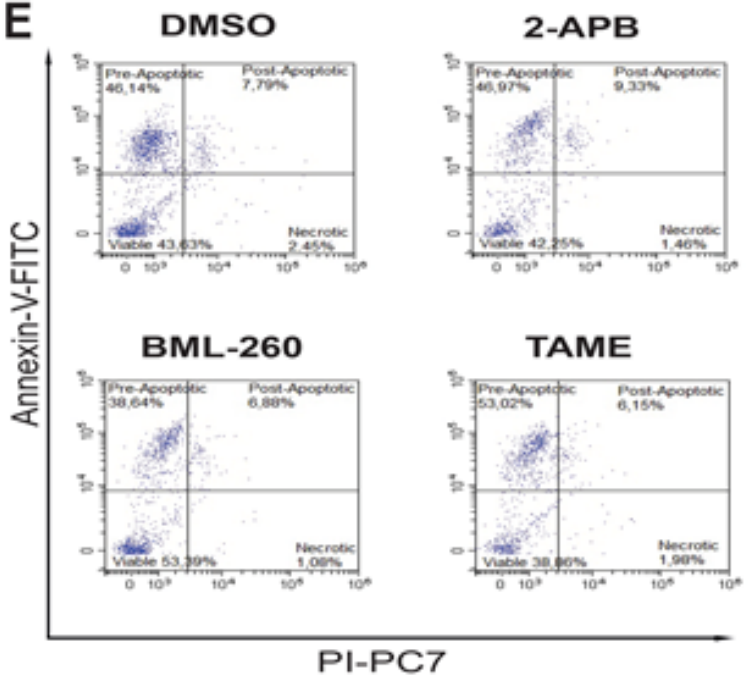

B BrdU+Cleaved PARP+ Cells

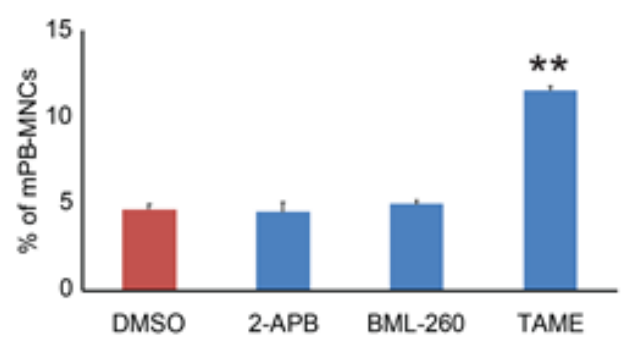

D H2AX+Cleaved PARP+Cells

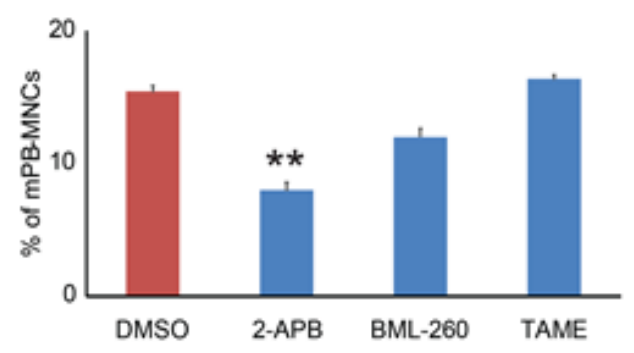

F Apoptosis Assay

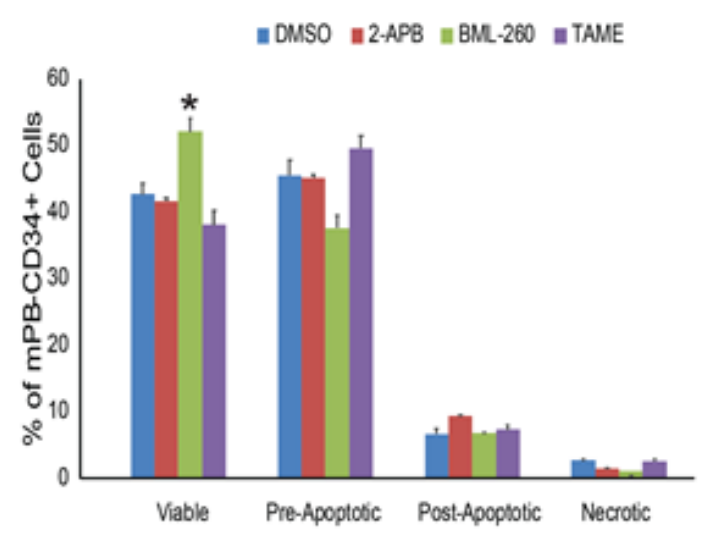

Figure 6

The viability, cytotoxicity and genotoxicity assessments of selected HSM expanded-mPB-HSPCs. (a) The results of viability assay post 7 days of treatments were shown with representative flow plots (left) and its quantification (right) compared to DMSO control. The quantification of genotoxicity was presented for (b) cycling cell ratio, (c) apoptotic cells and (d) DNA damage in cycling HSPCs and (e) the relation of DNA damage and apoptosis on the expanded cells compared to DMSO control. (f) The cytotoxicity assay performed post $72 \mathrm{~h}$ of treatment were shown for representative flow plots (left) and its quantification (right) and compared to DMSO control. $(n=3, * p<0,05, * * p<0,01)$. 


\section{A Analysis of key CDKI gene expressions}

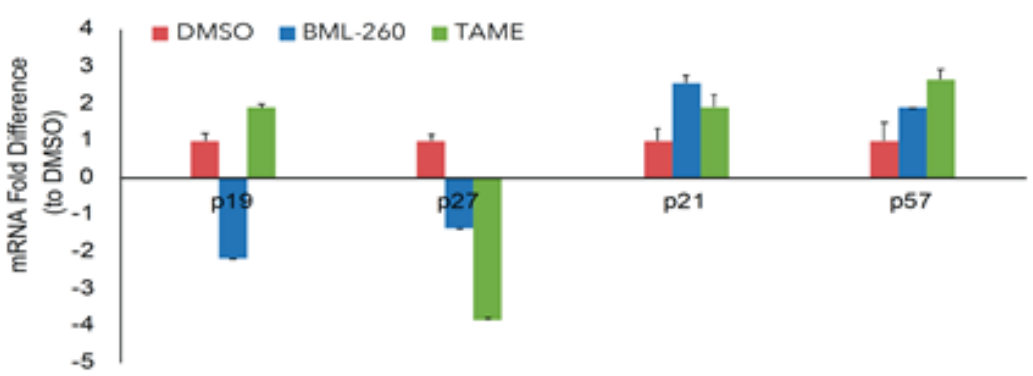

\section{B S-phase and HDR gene expression}

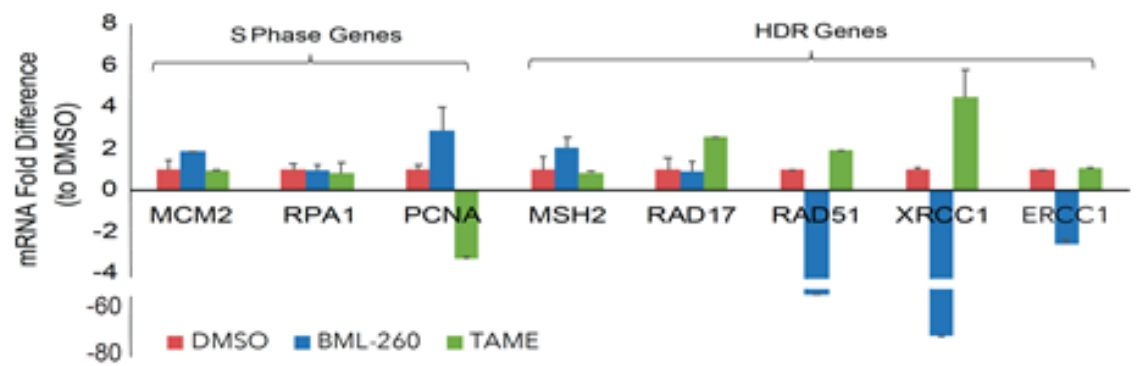

C Relavant target gene expression

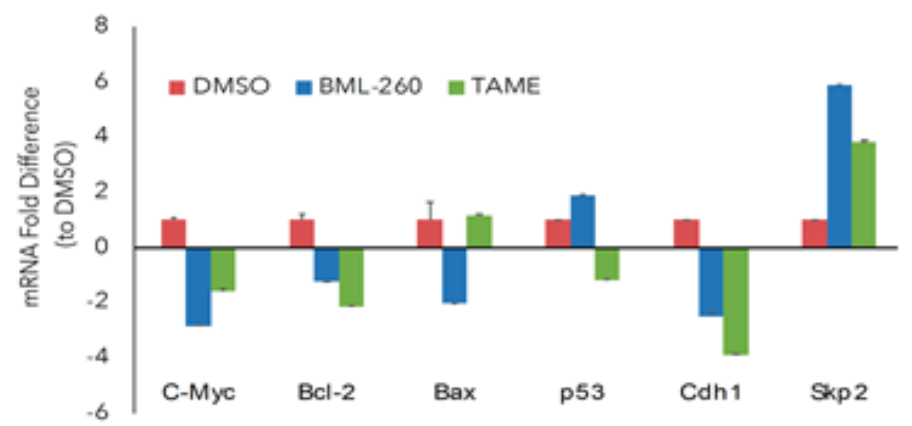

Figure 7

The gene expression analysis of HSM expanded-mPB-HSPCs. Real-time PCR was performed for determination of expression changes in CDKI genes (a), S-phase and HDR related genes (b) and BML-260 and TAME inhibitors related genes (c) compared to DMSO post 7 days of treatments. $\left({ }^{*} \mathrm{p}<0,05,{ }^{*} \mathrm{p}<0,01\right)$. 


\section{A}

huCD34+

isolation

HSC expansion and Retroorbital injection into SCID mice

Analysis of huCD45+ cells by flow cytometry

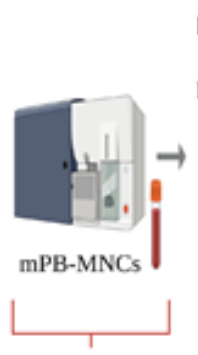

Day 0

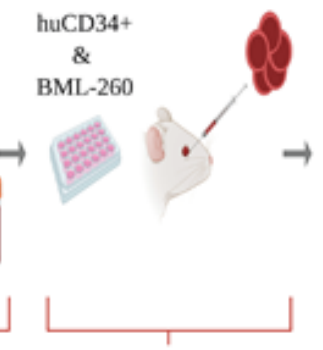

Day 7
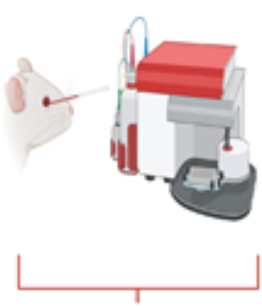

Days 30-150

B Repopulation analysis post 4 weeks of transplantation

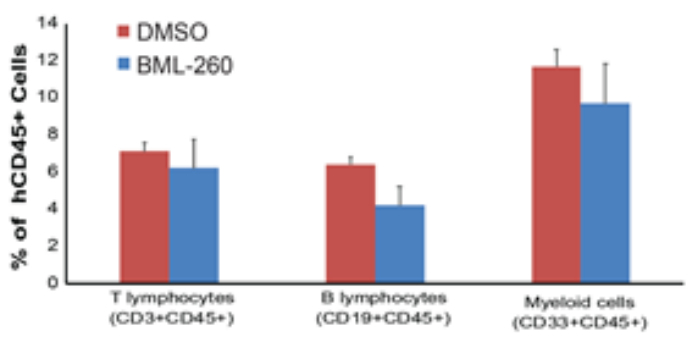

C Repopulation analysis post 20 weeks of transplantation

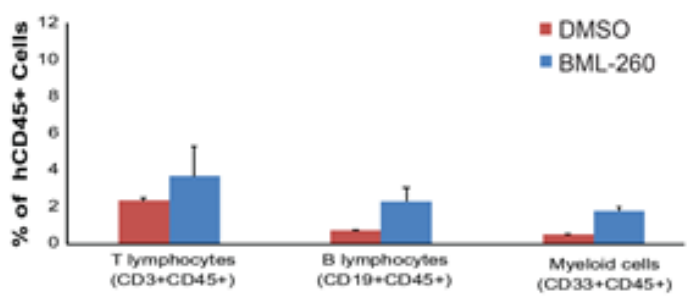

E Human cell content post xenotransplantation

D Human derived hematopoietic stem cell content post 20 weeks of transplantations
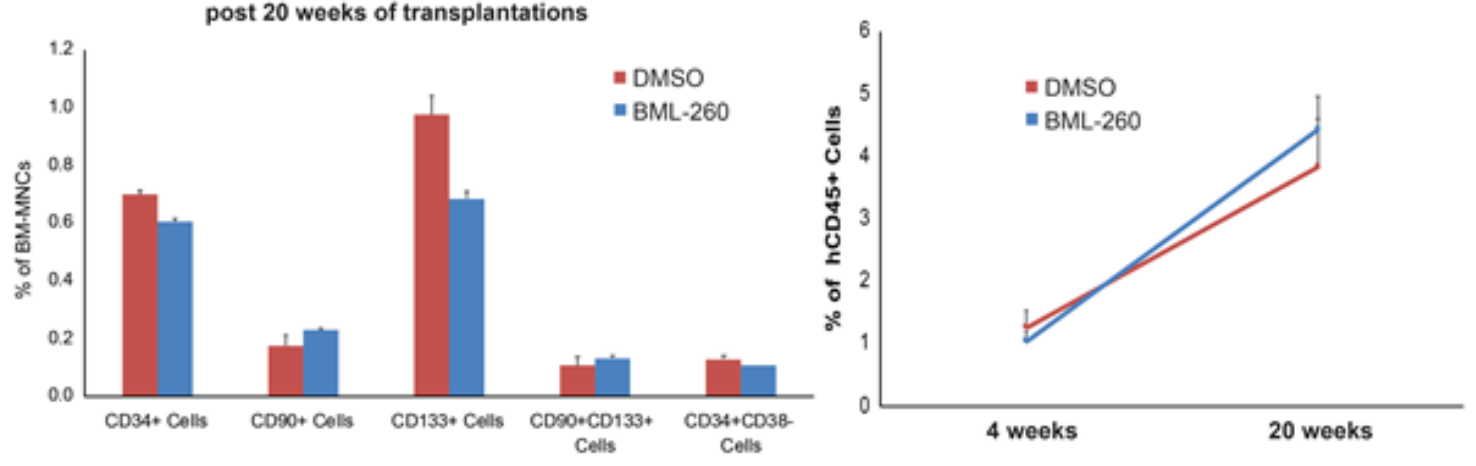

\section{Figure 8}

In vivo repopulation analysis of BML-260 expanded HSCs. (a) The experimental design about xenotransplantations. Repopulation analysis in the peripheral blood after (b) 4 and (c) 20 weeks. Repopulated HSCs were quantified based on the percentage of human CD45+ cell population for $\mathrm{T}, \mathrm{B}$ lymphoid and myeloid cells in comparison with DMSO group of treatments in mice $(n=4)$. The results of $(d)$ donor-derived hematopoietic cell content in BM counterparts $(n=6)$. (e) Analsis of circulating human CD45+ cells for STrepopulation (4 weeks) and LT-repopulation (20 weeks) $(n=4) .\left({ }^{*} p<0,05,{ }^{* *} p<0,01\right)$. 
A
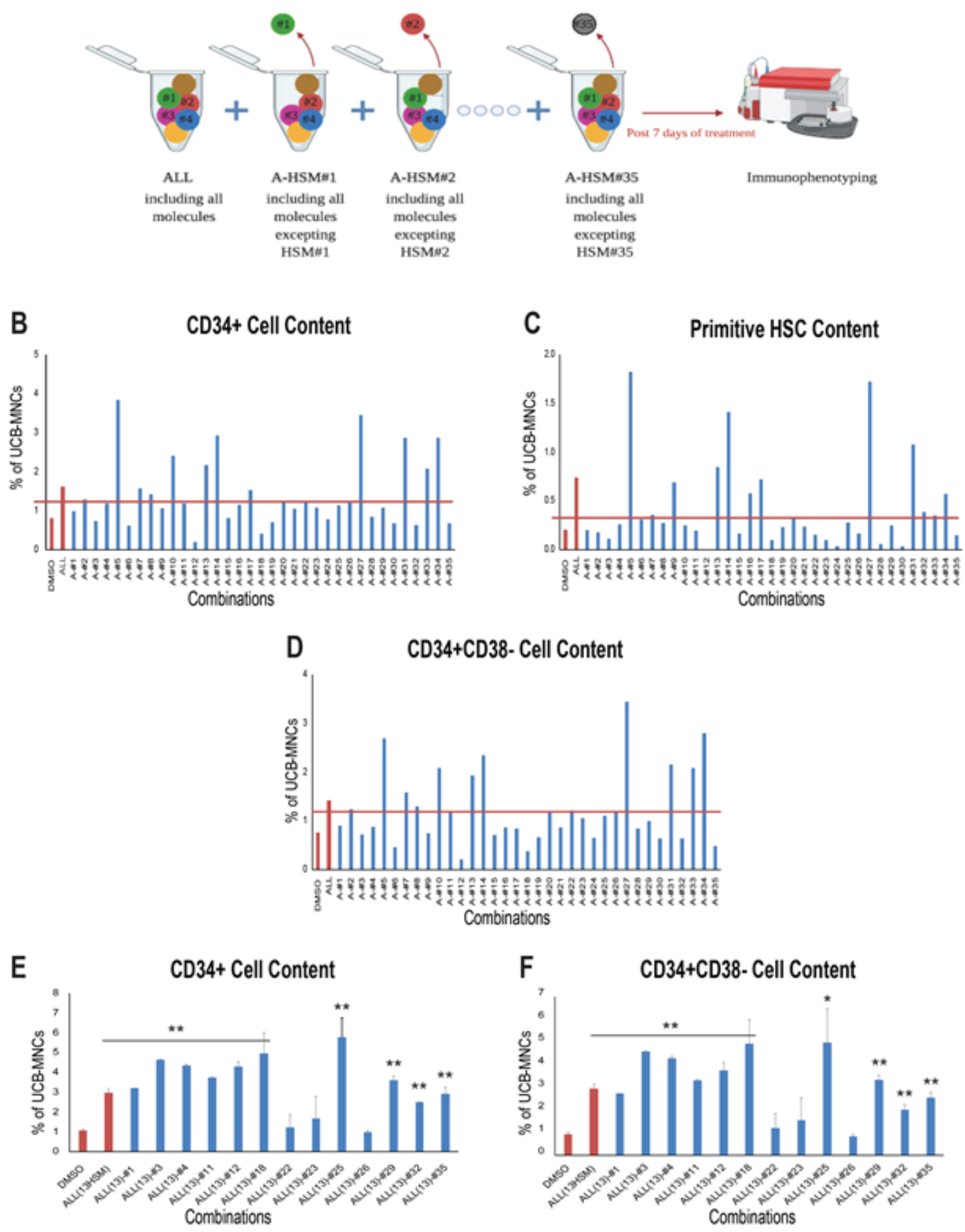

\section{Figure 9}

Development of HSM cocktail for human UCB-HSC expansion. (a) The strategy for HSM coctail experimental design. The essential HSMs were determined by the elimination of HSMs that resulted in an increased cell number more than 1.5 fold in the absence from $36 \mathrm{HSM}$ mixture compared to DMSO control. The results of 0.1 Im dose was shown for (b) CD34+ cell, (c) primitive, and (d) CD34+CD38- HSC contents. In the second step, 13 essential HSMs that are needed for HSC expansion were evaluated by the same way compared to ALL(13) (13 HSMs containing mixture) control. The results of $0.01 \mathrm{\square m}$ dose that worked well, was representatively shown for (e) CD34+ (left) and (f) CD34+CD38- cell contents (right). ( $n=3 ; * p<0,05, * \star p<0,01)$. 

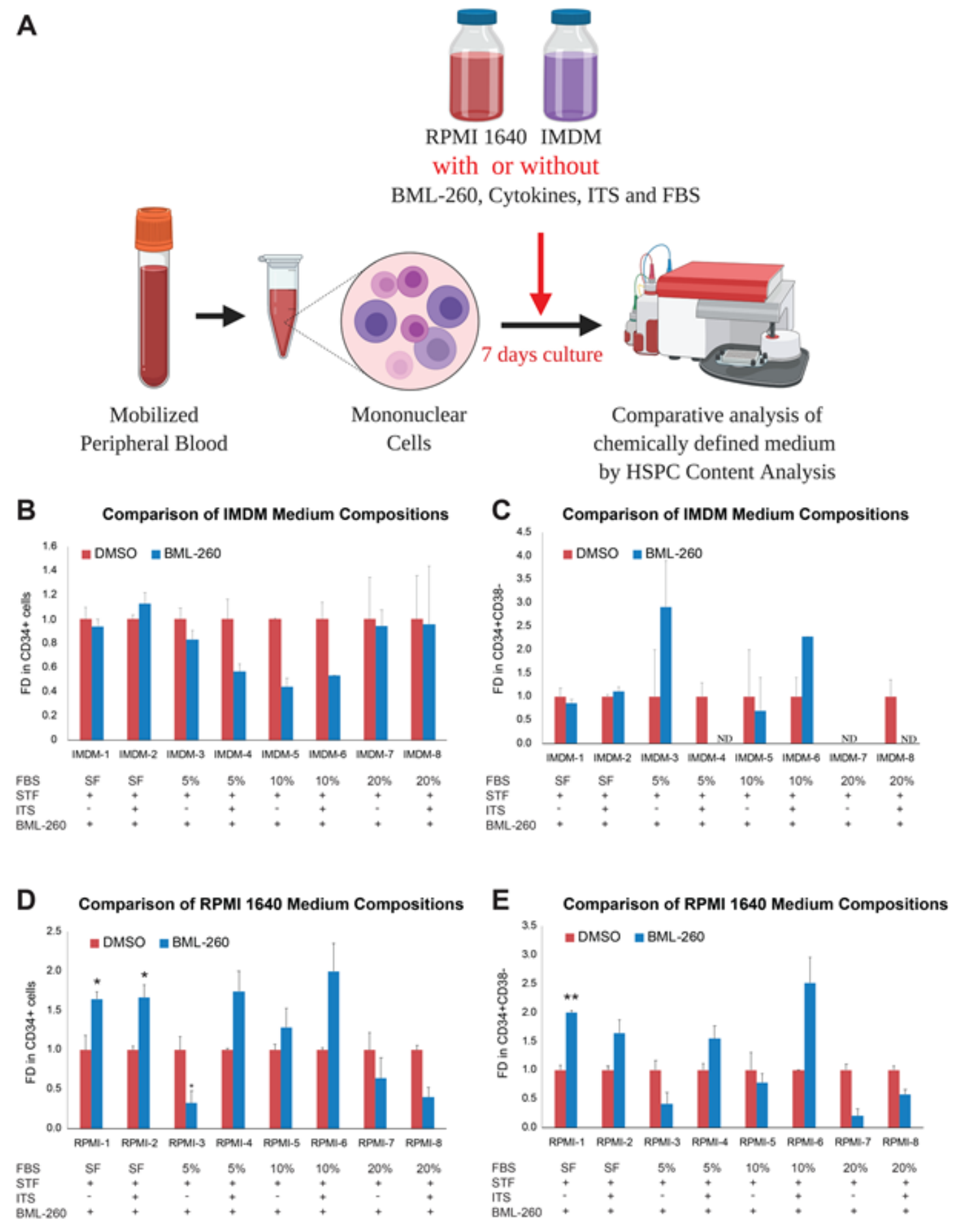

Figure 10

Comparative analysis of chemically-defined HSC expansion media supplemented with identified HSM and supplements. (a) The scheme of experimental study. The analysis of (b) CD34+ and (c) CD34+CD38populations in IMDM 1640 basal medium and its varying compositions. The analysis of (d) CD34+ and (e)CD34+CD38- populations in RPMI basal medium and its varying compositions. FBS: Fetal bovine serum, SF: Serum-free, STF: SCF, TPO, FL3-L cytokines, ITS: Insulin-Transferrin-Selenium.

\section{Supplementary Files}


This is a list of supplementary files associated with this preprint. Click to download.

- Graphicalabstract.jpg

- SupplementaryFiguresandlegendsFKvs3.docx 